\title{
Agôn
}

Revue des arts de la scène

HS 1 | 2011

Mettre en scène l'événement

\section{Témoigner pour le réel}

\section{Barbara Métais-Chastanier}

\section{(2) OpenEdition}

\section{Journals}

Édition électronique

URL : http://journals.openedition.org/agon/1787

DOI : 10.4000/agon. 1787

ISSN : 1961-8581

\section{Éditeur}

Association Agôn

\section{Référence électronique}

Barbara Métais-Chastanier, «Témoigner pour le réel 》, Agôn [En ligne], HS 1 | 2011, mis en ligne le 05 septembre 2011, consulté le 17 avril 2020. URL : http://journals.openedition.org/agon/1787 ; DOI : https://doi.org/10.4000/agon.1787 


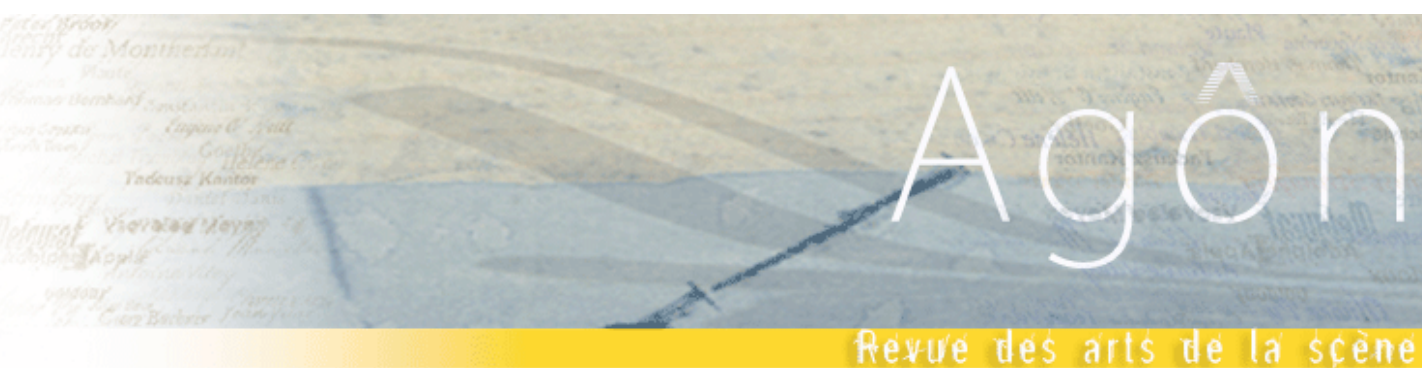

\section{Témoigner pour le réel}

\section{Barbara MÉTAIS-CHASTANIER}

À l'occasion de la mort de Ben Laden, mort sans corps et sans image, dont on pourrait rapidement conclure qu'elle fût donc sans preuve, deux images ont occupé, à quelques jours d'intervalle, les journaux d'une bonne partie de la planète : la première montre Barack Obama et son équipe rapprochée, réunis dans la « situation room » de la Maison Blanche, pour suivre en direct le déroulement de l'opération Geronimo alors en cours à Abbottabad - la disposition des corps, des regards, tous accrochés à ce point du hors-champ, bustes en avant, et la géographie des visages singulièrement disposés, font que tout converge vers ce point absent, zone aveugle de la composition, qui est elle-même une image, celle où l'événement s'est comme retiré. L'autre, parue peu de temps après, tirée d'une vidéo et soumise à de franches critiques et à de non moins francs soupçons, montre Ben Laden de dos - ou du moins un vieillard qu'on a décidé de nous faire prendre pour tel, sans doute pour orchestrer les conditions de possibilité d'un sabotage idéologique : on sape l'image (la copie) plutôt que de montrer le corps (la preuve) - bonnet vissé sur la tête, couverture remontée sur les épaules, profil perdu et comme déjà retiré, cherchant à apercevoir sur l'écran qui lui fait face le produit de ce qu'il a patiemment élaboré pendant vingt ans. Une image (l'écran) et son retrait (le dos).

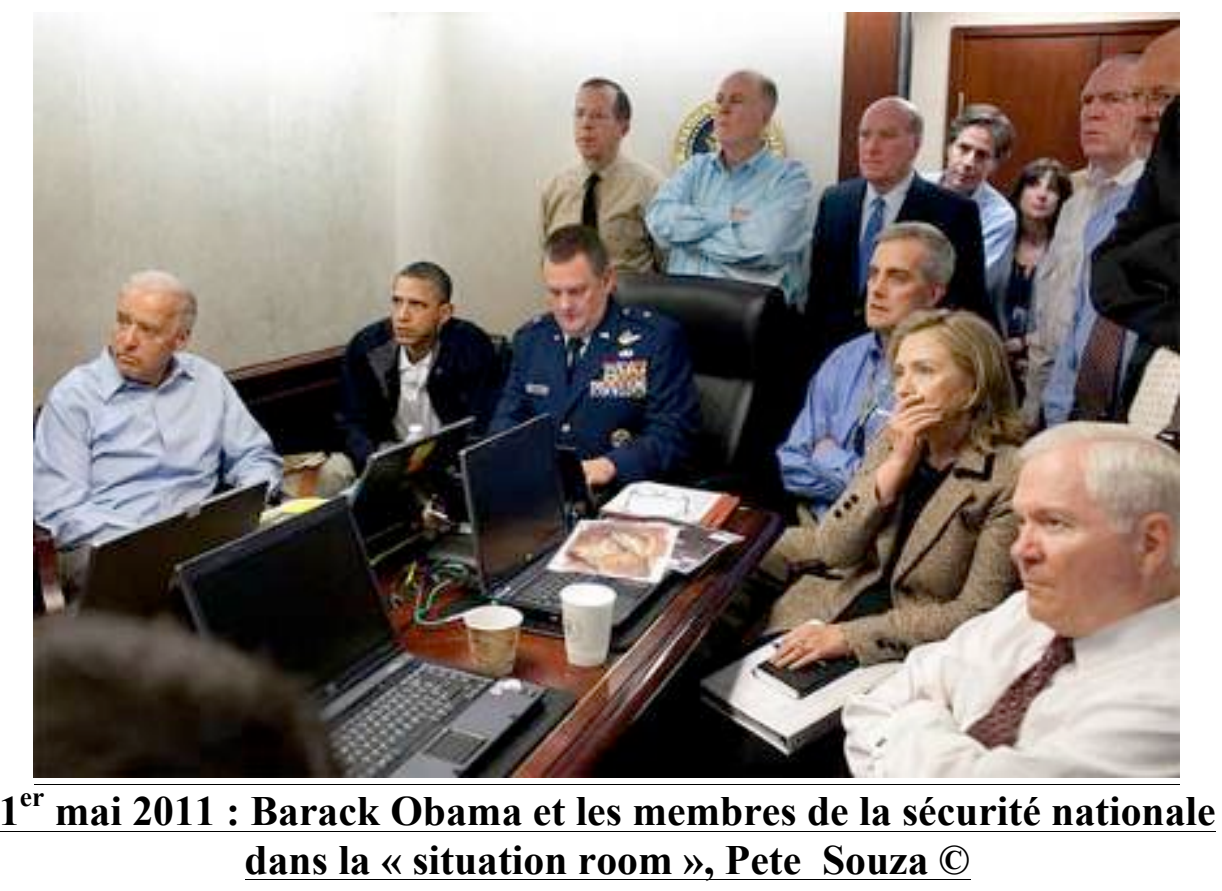




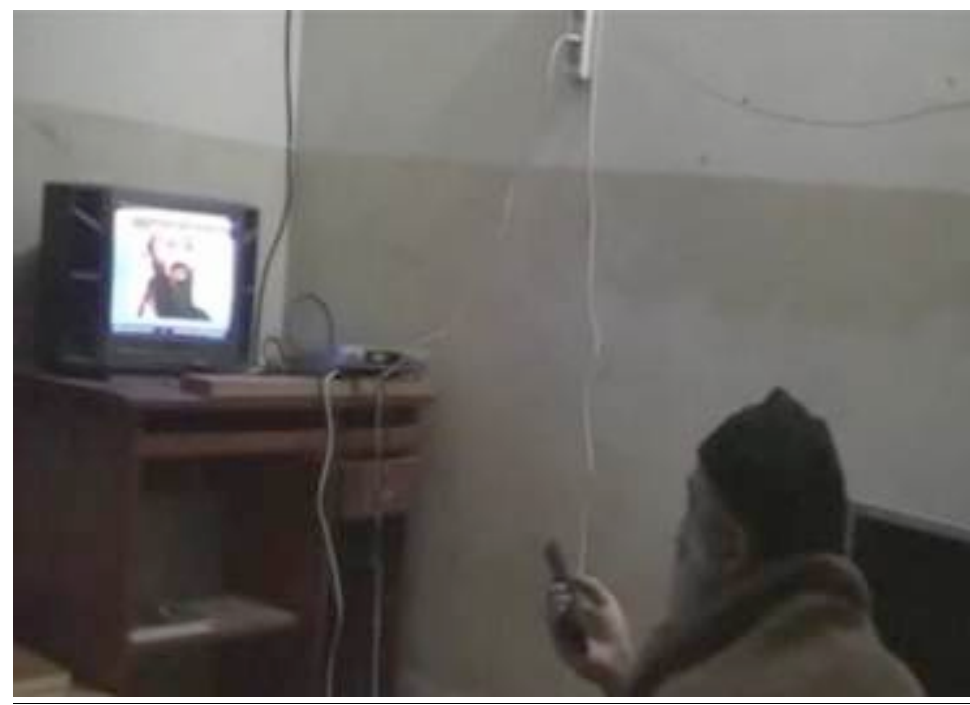

\section{Capture d'écran d'une vidéo saisie dans la propriété d'Oussama Ben Laden et diffusée par le Pentagone le 7 mai 2011.}

Ces deux photographies, Marie-José Mondzain et Christian Salmon les rapprochent également dans deux articles publiés dans Le Monde du 14 mai 2011. Si la première insiste sur la stratégie de colonisation de l'image mise en place par Ben Laden', le second s'appuie, lui, sur le «dispositif de vision » que mettent en scène ces photographies : "Ces photos ne montrent rien d'autre qu'un dispositif où règne le pur "simulacre", qui n'est plus de l'ordre du spectaculaire au sens strict, puisque à proprement parler il n'y a rien à voir, mais spectral, en ce qu'il installe une relation triangulaire où chacun regarde l'autre en train de voir ce qui n'est pas visible ${ }^{2}$. " N'y a-t-il « à proprement parler » rien à voir ? Là où Christian Salmon choisit de mettre l'accent sur le retrait, ce qui reste à jamais perdu dans la relation à l'événement, relégué au rang de simulacre par les médiations enchâssées, je préfèrerais m'attarder dans cet article sur ce « dispositif de vision », très justement remarqué par l'auteur, en revenant sur la configuration particulière qui enchaîne le réel à sa restitution, l'événement à sa représentation et à sa réception, à travers la présence du témoin.

L'entrée du témoin substitue à la relation binaire et frontale de l'observateur et de la chose vue, la « relation triangulaire », réflexive, médiatisée d'un regard passé de main en main et, ici, d'image en image. Qu'y-a-t-il à voir dès lors que l'on voit «voir»? N'y a-t-il «à proprement parler rien à voir » sinon une image vide qui donne l'absence par la présence d'un regard qui fouille le hors-champ ? N'y a-t-il rien de l'événement en persistance dans le témoin et dans la manière dont le réel filtre à travers lui ? Avant même d'entrer dans les enjeux qui travaillent les scènes contemporaines - qui se distinguent par le recours récurrent au témoin et au témoignage -, remarquons seulement combien ce dispositif spéculaire fait fond sur quelque chose de proprement théâtral, si tant est que l'on veuille bien s'en rapporter à la seule étymologie, épuisée à force d'être reprise, mais signifiante ici pour la seule raison que le theatron était d'abord le lieu d'où l'on peut voir, c'est-à-dire l'espace depuis lequel on assistait en témoin, autrement dit en tiers, au déroulement d'une action. Le témoin (testis composé du radical tes, originaire de tres, trois $^{3}$ ) - comme le précise Arnaud Dulong en

\footnotetext{
${ }^{1}$ Marie-José Mondzain, «Il est plus facile de tuer un homme que d'effacer son image », Le Monde, 14 mai 2011.

${ }^{2}$ Christian Salmon, « Un crime parfait », Le Monde, 14 mai 2011.

${ }^{3}$ Émile Benveniste précise dans Le Vocabulaire des institutions indo-européennes (Paris, Minuit, 1969) que témoin dérive de tertius, qui désignait les tierces personnes chargées d'assister à un contrat oral et qui étaient habilitées à certifier cet échange.
} 
s'appuyant sur une étude réalisée par Benveniste ${ }^{4}$ - était, en droit romain, le tiers, troisième ou tierce personne qui assistait à un procès en tant que soutien à chacune des parties.

C'est sur ces «appareillages du vrai $^{5}$ » qui occupent les scènes contemporaines que je souhaiterais revenir dans cet article en m'attardant sur le dispositif du témoin (quelqu'un regarde quelque chose), et corrélativement du témoignage (quelqu'un fait récit de quelque chose qu'il ne m'a pas été donné de voir), et du type de réception impliquée par ces situations (quelle expérience du réel ou de la fiction s'instaure dans le sertissage des regards et des voix ? Suis-je moi-même témoin, observateur, juge ou spectateur de ce qu'il m'est donné à entendre ou à voir?). Une fois détaillée la récurrence et ses lignes particulières, reste à comprendre les raisons de cette échappée contemporaine vers l'actualité, le document et l'événement : que disent ces stratégies d'authentification? De quoi témoigne le recours au témoin ? De quoi est-il le signe? Ambition sans doute trop vaste pour un simple article mais qu'il faudra tenter de garder présente à l'esprit car tel est aussi l'étrange privilège du témoignage aujourd'hui (comme dispositif esthétique mais aussi comme acte discursif) que d'être le symptôme d'une carence qui travaille le sujet comme la société - lacune du réel à l'endroit même qu'il devrait occuper et qui se traduit par une inflation du régime de la preuve, de l'authentique et de la caution - mais également l'outil de sa contestation, les conditions de son propre dépassement.

Le désir d'arpenter ce vaste territoire m'aura été soufflé par l'écriture de Michel Vinaver, 11 septembre 2001 bien sûr - pour la récurrence et la place donné au témoignage qui invite celui qui le met en scène à trouver les conditions de cette prise de parole ${ }^{6}$ - mais également le projet D'un 11 septembre à l'autre lui-même, qui dans sa première lancée associait à Arnaud Meunier Rachid Ouramdane, chorégraphe qui traverse depuis plusieurs années les usages et les conséquences d'une «poétique du témoignage ». Étrange conjonction donc qui venait répéter, reprendre et prolonger une tentation du témoignage à l'œuvre dans les écritures contemporaines, tentation à laquelle je m'efforce ici de donner quelques traits.

\section{$E t$, très vite, il n'y eut plus rien à voir}

«Un jour, il y a bien longtemps, je tombai sur une photographie du dernier frère de Napoléon, Jérôme (1852). Je me dis alors, avec un étonnement que depuis je n'ai jamais pu réduire : "Je vois les yeux qui ont vu l'Empereur." " Roland Barthes, La Chambre claire.

Le tiers, c'est lui aussi qu'Olivier Culmann, photographe du collectif Tendance Floue, va chercher, et sur lui qu'il braque son objectif, que celui-ci soit mis en présence du réel, comme dans la série Autour, New York 2001-2002 qui s'attache aux visages des passants regardant les ruines du World Trade Center, ou qu'il se soit absenté dans l'image replète livrée par la

\footnotetext{
${ }^{4}$ Arnaud Dulong, Le Témoin oculaire. Les conditions sociales de l'attestation personnelle, Paris, EHESS, 1998, p. 43 .

${ }^{5}$ Noëlle Renaude et Barbara Métais-Chastanier, Accidents, à paraître.

${ }^{6}$ Robert Cantarella, «Ce qui traîne autour du lieu», Agôn [En ligne], Dossiers, HS n 1 : Mettre en scène l'événement, 11 septembre 2001 de Michel Vinaver, mis à jour le : 30/08/2011, URL : http://w7.ens$\underline{\text { lsh.fr/agon/index.php?id=1783 }}$
} 
télévision, avec la série Watching $T V$, où le corps disparaît dans la contemplation du petit écran, s'y abandonne autant qu'il s'y appuie. L'exposition " Watchers ${ }^{7}$ », présentée à Paris du $1^{\mathrm{er}}$ avril au 11 juin 2011 au pavillon Carré de Baudoin, met en regard ces deux séries qui sont d'abord deux formes de déplacement : Olivier Culmann occupe résolument le terrain du horschamp, fait le choix du pas de côté par rapport à l'événement et à la brûlante actualité. Avec la série Autour, New York 2001-2002, c'est dans les pourtours qu'il va chercher les traces de ce «major event ${ }^{8}$ » que furent les attentats du 11 septembre 2001, " événement absolu, selon Jean Baudrillard, la "mère" des événements, [...] l'événement pur qui concentre en lui tous les événements qui n'ont jamais eu lieu'. » Ce léger écart, cette volte-face, qui lui font tourner le dos à l'endroit vers lequel convergent pourtant tous les regards, permet à Olivier Culmann - et plus largement à celui qui fait entrer le témoin sur scène et opte pour le contre-champ de tenir de front l'impression et l'événement : regarder quelqu'un voir, c'est aussi partir en quête d'une vérification. Que font-ils tous ces gens dont le regard fouille les décombres du World Trade Center? De quelle attestation, de quelle certitude sont-ils en quête ? Et lui, le photographe, que saisit-il ? que donne-t-il à voir?

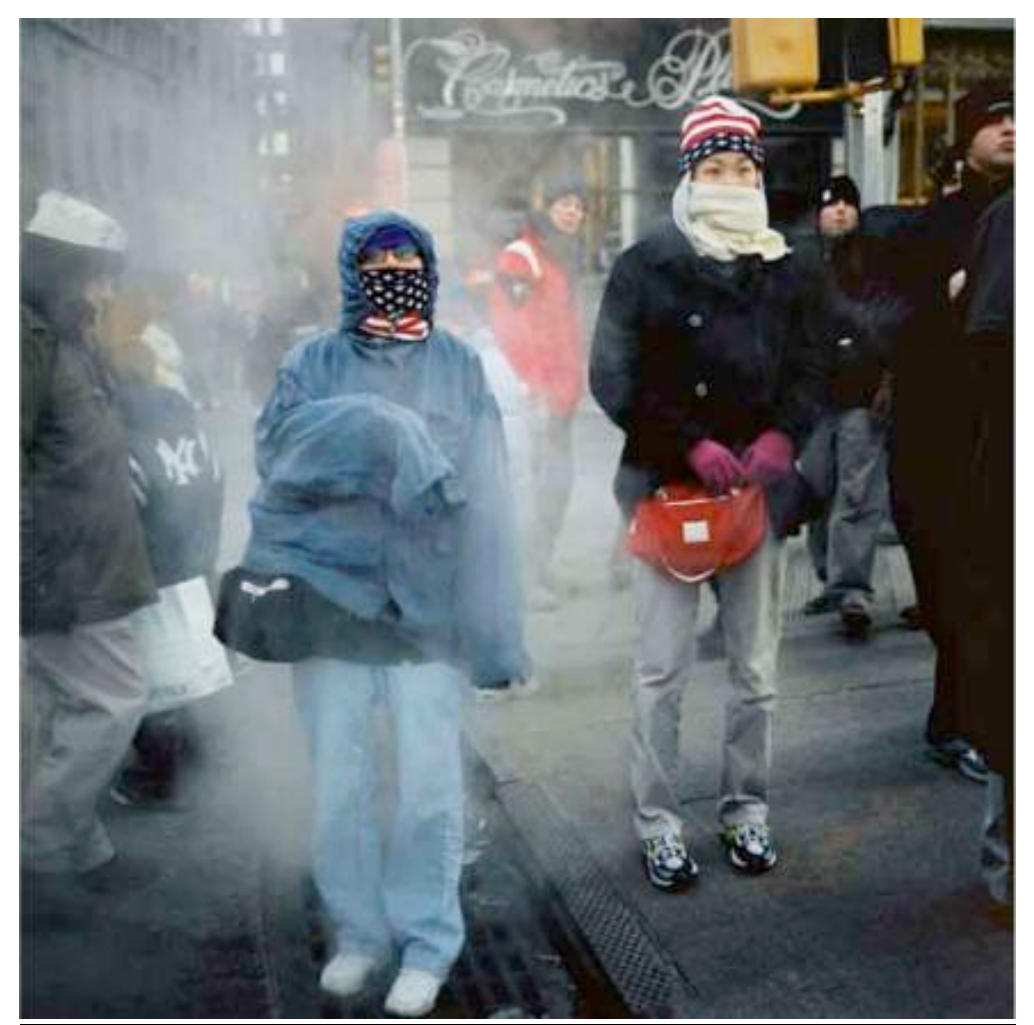

Olivier Culmann (C, « Autour. New York. 2001-2002 »

Prendre en photo le tiers, le témoin, celui qui doute, s'interroge et observe, c'est saisir le visage dans le geste de vérification, voler un doute et le donner comme certitude, ouvrir l'espace oblique de l'événement et de son adresse. Il y a l'en soi de l'événement, puissance

\footnotetext{
${ }^{7}$ Olivier Culmann, exposition « Watchers » présentée au pavillon Carré de Baudouin du $1^{\text {er }}$ avril au 11 juin 2011 à l'occasion des vingt-ans du collectif Tendance Floue.

${ }^{8}$ L'expression est employée à plusieurs reprises par Giovanna Borradori in Jacques Derrida, Jürgen Habermas, Le "concept" du 11 septembre, Dialogues à New York (octobre-décembre 2001) avec Giovanna Borradori, Paris, Galilée, 2004. Voir par exemple la première question qu'elle adresse à Jacques Derrida : « Le 11 septembre nous a donné l'impression d'être un major event, un des événements historiques les plus importants auquel nous assisterons dans notre vie, particulièrement pour ceux d'entre nous qui n'ont pas vécu la guerre mondiale. Qu'en pensez-vous? », p. 133

${ }^{9}$ Jean Baudrillard, L'Esprit du terrorisme, Paris, Galilée, 2002, pp.9-10.
} 
neutre du «c'est ça », évidence de la chose arrivée, survenue : «Le on de l'événement pur où il meurt comme il pleut ${ }^{10} »$, écrit Deleuze dans Logique du sens (1969). C'est la force d'effraction de ce qui arrive, intenable, impossible et pourtant réalisé, inattendu et pourtant réintégrable rétrospectivement dans une chaîne de raisons et d'effets, "part que [son] accomplissement ne suffit pas à réaliser, écrit encore Deleuze dans Dialogues (1977), un devenir en lui-même qui ne cesse à la fois de nous attendre et de nous précéder comme une troisième personne de l'infinitif, une quatrième personne du singulier ${ }^{11}$. " Mais il y a aussi, imperceptiblement coulée en lui, ce qui l'atteste pour ce qu'il est: l'impression de l'événement. S'il y a un en soi de l'événement, il n'y a pas d'événement en soi, d'événement qui ne soit pas relatif à ses conditions d'apparition, à son temps de surgissement et à la façon dont il se présente à la conscience, dont il lui arrive, et lui tombe dessus.

Dans les premières minutes de son dialogue avec Giovanna Borradori, qui a pour objet l'étude et la compréhension du « concept» du 11 septembre 2001, Jacques Derrida revient sur cette double nature de l'événement, visage déplié de la chose en soi et de son impression, Janus aux deux faces qui constitue pourtant et malgré tout une seule et même figure :

Indéniablement, dirait littéralement un empiriste du XVIII ${ }^{\mathrm{e}}$ siècle, il y a eu là une "impression», et l'impression de ce que vous appelez, non fortuitement, en anglais, un « major event ». [...] Qu'elle soit justifiée ou non, cette « impression», elle-même est en soi un événement, il ne faut jamais l'oublier, surtout quand, de façon certes différenciées, elle est un effet proprement mondial. L' «impression» ne se laisse pas dissocier de tous les affects, des interprétations, des rhétoriques qui l'ont à la fois réfléchie, communiquée, «mondialisée », mais aussi et d'abord formée, produite, rendue possible. L' «impression » ressemble alors à la «chose même » qui l'a produite. Même si ladite " chose » ne s'y réduit pas. Si donc l'événement même ne s'y réduit pas. L'événement est fait de la « chose » même (ce qui arrive) et de l'impression (elle-même à fois « spontanée » et "contrôlée ») que ladite « chose » donne, laisse ou fait ${ }^{12}$.

Si l'événement ne se réduit pas à l'impression qu'il produit, l'impression n'en constitue pas moins un événement à elle seule : elle fait événement. Quelque chose passe dans l'image, dans le film, dans le temps qui est, en lui-même, une effraction. Et dès lors, choisir de s'intéresser à cette impression, décider de se concentrer sur cette part infime du réel, celle du visage, du regard et du corps, qui est mise en présence de ce qui a lieu, a eu lieu, ou s'annonce par le détour de celui qui le reçoit, c'est aussi tenter d'échapper au régime de sidération produit par l'image-choc, celle qui se donne comme pleine, et qui pour cette même raison n'est qu'un support idiot. C'est encore cette bêtise que Barthes relevait quand il écrivait en 1961 dans Le Message photographique: «La photographie traumatique (incendies, naufrages, catastrophes, morts violentes, saisis "sur le vif") est celle dont il n'y a rien à dire : la photo-choc est par structure insignifiante : aucune valeur, aucun savoir, à la limite aucune catégorisation verbale ne peuvent avoir prise sur le procès institutionnel de la signification $^{13}$. »Image repue, complète, sans appel ni entêtement, la photo-choc ne laisse aucune place à celui qui la regarde.

\footnotetext{
${ }^{10}$ Gilles Deleuze, Logique du sens, Paris, Minuit, 1969, p. 178.

${ }^{11}$ Gilles Deleuze et Claire Parnet, Dialogues, Paris, Flammarion, coll. « Champs », 1996, p. 79.

12 Jacques Derrida, Jürgen Habermas, Le «concept» du 11 septembre, Dialogues à New York (octobredécembre 2001) avec Giovanna Borradori, op. cit., pp. 136-137.

${ }^{13}$ Roland Barthes, « Le Message photographique », in Communications, n¹, Paris, Seuil, 1961, p. 137.
} 


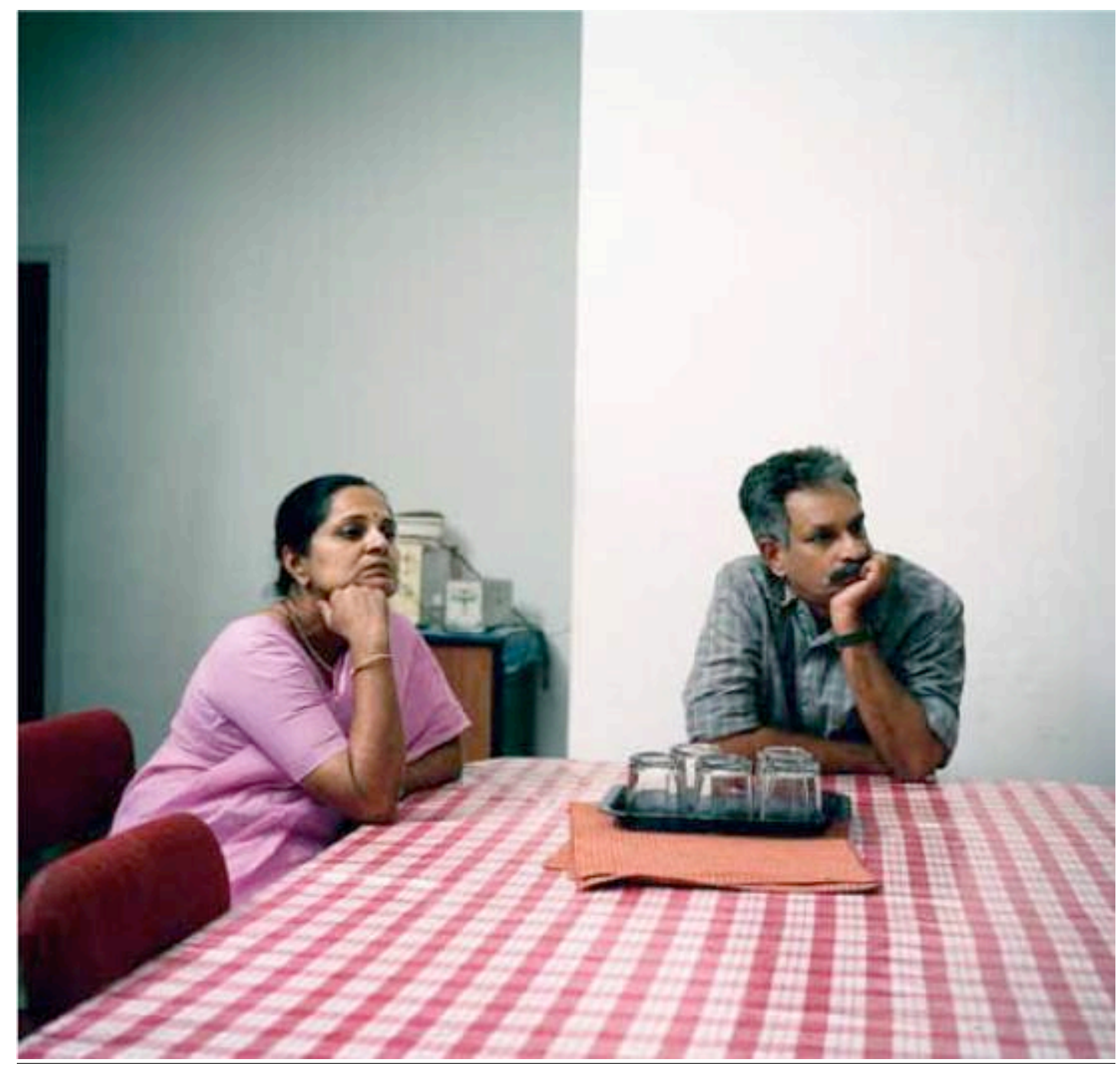

Olivier Culmann ${ }^{C}$, « Watching TV»

C'est travailler à l'envers de « ce monde sans hors-champ ${ }^{14} »$, pour tenter de réinstaurer de l'absence et du divers dans le traitement de l'événement, que d'aller chercher dans les pourtours de l'évidence. Le témoin offrirait donc l'appel, une ventilation du visible par le retrait, un espace d'appréhension détourné, oblique et second. Car le 11 septembre 2001, s'il fut l'événement le plus photographié de l'histoire et celui qui se singularise par une couverture médiatique jamais égalée, fut surtout donné à voir à travers une variété d'images très réduite. C'est ce qu'analyse remarquablement Clément Chéroux, historien de la photographie et conservateur au Centre Pompidou, dans une récente étude, Diplopie: L'image photographique à l'ère des médias globalisés : essai sur le 11 septembre $2001^{15}$. Son enquête, appuyée sur l'analyse des couvertures de quatre-cent journaux américains parus les 11 et 12 septembre 2001, révèle qu'en réalité cette large production peut se rapporter à une série de six images-types : explosion de la tour sud $=41 \%$; nuage de fumée au-dessus de Manhattan alors que les tours brûlent $=17 \%$; ruines de Ground Zero $=14 \%$; image de l'avion avant l'impact $=13,5 \%$; scènes de panique dans les rues de Manhattan $=6 \%$; image des trois pompiers hissant le drapeau américain $=3,5 \%$. La catastrophe telle qu'elle nous a été donnée à voir par les médias bégaie sans cesse autour des mêmes images, répétant le scénario

\footnotetext{
${ }^{14}$ Marie-Josée Mondzain, Homo Spectator, Paris, Bayard, 2007, p. 91.

${ }^{15}$ Clément Chéroux, Diplopie. L'image photographique à l'ère des medias globalisés. Essai sur le 11 septembre 2001, Cherbourg-Octeville, Le Point du jour, 2009.
} 
d'un désastre où l'humain n'a que peu de part: "Comme par métonymie, écrit Clément Chéroux, c'est la souffrance du bâtiment qui domine : le World Trade Center frappé, blessé, et finalement anéanti ${ }^{16}$. » Aller chercher le visage, l'impression avant même qu'elle ne se donne comme expression, témoignage ou récit, est une façon d'élaborer un autre imaginaire du visible, un geste discret, en prise sur l'intime et le singulier, qui tente d'aller œuvrer ailleurs que dans l'écholalie. C'est cette carence du visible - succédant à la pléthore d'images - qui retient d'ailleurs Olivier Culmann dans le texte de présentation de sa série Autour :

Les premières images des attentats du 11 septembre 2001 me sont parvenues, comme à des millions d'autres personnes, dans l'irréalité la plus totale. Á travers l'écran de télévision, la dimension de la catastrophe restait confinée dans l'ordre du cinématographique, de la fiction, de l'impensable. Il fallait y aller pour tenter de réaliser l'événement. Au début, il y avait encore quelque chose : des pans de murs continuaient à fumer. Et, très vite, il n'y eut plus rien à voir. Mais les gens venaient malgré tout et regardaient le vide. Eux-mêmes essayaient de vérifier l'anéantissement ${ }^{17}$.

« Très vite, il n'y eut plus rien à voir »: le visible s'étant comme retiré, ne restait plus que le désir de chercher à voir pour tenter de comprendre - et s'efforcer de trouver le moyen de saisir cette incertitude au moment où elle passait. "Réaliser l'événement », c'était donc tout autant en prendre la mesure que l'accomplir et l'effectuer - restituer son impression (ce qu'il nous fait) et sa représentation (ce que l'on fait de lui) à travers ses seules traces. S'intéresser au témoin, au tiers, c'est en effet choisir une image pauvre (« à proprement parler il n'y a rien à voir », écrivait Christian Salmon), manquante et restituant son absence : les quelques vingttrois clichés que comptent la série Autour exposée au Pavillon du Carré de Baudouin n'offrent au regard qu'un autre regard, regard sans cible, qui ne fait sens que par ce qu'il y a précisément autour : la légende qui ne vaut ici que par sa valeur indicielle, le lieu, la date New York, septembre 2001, décembre 2001 et février 2002 - suffisent à faire image. La photographie devient alors promesse d'un autre visible, témoin d'une autre image, grosse d'un réel qui se refuse au cadre : elle se désigne tout à la fois comme présence et absence.

Il est intéressant de remarquer que Robert Cantarella ${ }^{18}$, metteur en scène de 11 septembre 2001 à Los Angeles en 2004, aura suivi le même trajet qu'Olivier Culmann dans son désir de venir attester de l'événement : parti à New York en 2004 avec le projet de faire un film sur le chantier de reconstruction et sur les ouvriers venus du monde entier « qui s'engagent au nom du nouveau nouveau monde ${ }^{19} »$, avec le désir aussi de trouver dans cette exploration une manière d'entrer dans la pièce qu'il traversa bientôt avec les élèves du CalArts, il en revient avec des visages - les expressions de passants, d'habitants, de riverains, de curieux de passage, autant d'inconnus, de touristes d'un jour qui composent un feuilletage d'expressions singulières toutes appuyées sur l'absence d'une image - celle du chantier et de Ground Zero. Dans Carrosserie ${ }^{20}$, en effet, la caméra fouille les regards de ceux qui cherchent à voir les traces de la catastrophe. Avant le témoignage, qui constitue comme un temps second, une reprise à nouveaux frais, et un déplacement dont il faudra détailler les modalités, il y a donc le temps du témoin, temps suspendu du regard silencieux, étonnement pour soi, sans intention ni

\footnotetext{
${ }^{16}$ Ibid., p. 24.

${ }^{17}$ Olivier Culmann, « Autour, New York 2001-2002», url=http://www.tendancefloue.net/ouverture fr.html

${ }^{18}$ Robert Cantarella, «Ce qui traîne autour du lieu», Agôn [En ligne], Dossiers, HS n 1 : Mettre en scène l'événement, 11 septembre 2001 de Michel Vinaver, mis à jour le : 30/08/2011, URL : http://w7.enslsh.fr/agon/index.php?id=1783

19 Robert Cantarella, texte de présentation de Carrosserie (2004), url= http://www.robertcantarella.com/index.php?/film/carrosserie/, consulté le 12/06/2011.

${ }^{20}$ Robert Cantarella, Carrosserie (2004), $26 \mathrm{mn}$, Grenade Productions, Paris. Url= http://vimeo.com/18968080, consulté le 11/06/2011.
} 
adresse, où c'est le corps qui, prenant la parole de court, occupe tout l'espace. L'enchâssement des regards sur lesquels glisse la caméra participe tout à la fois d'une mise à distance (on étouffe l'obscénité du visible en le donnant comme regardable) et lui offre de l'intérieur le crédit d'une attestation (un spectateur vient réaliser dans l'image que ce qu'on me donne à voir est visible). Ouvert sur ces visages aspirés, et comme peuplés par des regards, le film habite avec le spectateur l'endroit-même de sa question : qu'est-ce que voir quand il n'y a rien ? Qu'est-ce que donner à voir un présent qui n'est plus ? Et comment regarder ce qui semble faire retraite ?

Les images de tiers et du témoin, où la chose n'est donnée qu'à travers la médiation d'une autre figure, interrogent le spectateur par ce qu'elles offrent et par ce qu'elles refusent, tout autant qu'elles questionnent cette place étrange et en retrait de l'observateur, de celui devant qui quelque chose arrive. Le titre choisit par Olivier Culmann pour réunir ses deux séries autour du regard est de ce point de vue assez éclairant : "Watchers » désigne tout à la fois le témoin et le spectateur, le guetteur, le veilleur, le gardien ou le surveillant voire l'expertobservateur, dressant ainsi la carte immense des rapports qui nouent le "watcher » au visible d'abord, à l'événement ensuite. Figure silencieuse, le «watcher» est, en effet, traversé de lignes d'activité et de passivité, en lui se répondent des positions contradictoires d'investissement dans le soin, le souci ou l'empathie (le garde-malade ou le veilleur), et de retrait, d'écart, d'une distance placée entre soi et le monde, que celle-ci opère sur le mode de l'attente (le guetteur), sur celui du signe et du savoir (Kremlin-watchers ; China-watcher) ou sur le mode du contrôle (le surveillant). La passivité n'est pas inhérente à la condition du spectateur, elle est une des modalités du regard et plus encore ce qu'on estime devoir être sa modalité, ainsi que le souligne Jacques Rancière dans Le Spectateur émancipé (2008) :

Qu'est-ce qui permet de déclarer inactif le spectateur assis à sa place, sinon l'opposition radicale préalablement posée entre l'actif et le passif? Pourquoi identifier regard et passivité, sinon par la présupposition que regarder veut dire se complaire à l'image et à l'apparence en ignorant la vérité qui est derrière l'image et la réalité à l'extérieur du théâtre ? Pourquoi assimiler écoute et passivité sinon par le préjugé que la parole est le contraire de l'action ? Ces oppositions - regarder/savoir, apparence/réalité, activité/passivité - sont tout autre chose que des oppositions logiques entre termes bien définis. Elles définissent proprement un partage du sensible, une distribution a priori des positions et des capacités et incapacités attachées à ces positions ${ }^{21}$.

Autrement dit, et la remarque vaut pour Carrosserie de Robert Cantarella, comme pour les deux séries de l'exposition Watchers d'Olivier Culmann, quelque chose dans le recours à la présence du témoin permet d'œuvrer à la restitution oblique de l'événement, d'échapper à l'obscénité du visible ou à son insondable distance. Le témoin, le tiers, rapproche et protège tout à la fois. Et ces démarches sont autant de points d'affirmation et de rappel que «l'acte de voir n'est pas l'acte d'une machine à percevoir le réel en tant que composé d'évidences tautologiques, ainsi que l'écrit Georges Didi-Huberman. L'acte de donner à voir n'est pas l'acte de donner des évidences visibles à des paires d'yeux qui se saisissent unilatéralement du "don visuel" pour s'en satisfaire unilatéralement. Donner à voir, c'est toujours inquiéter le voir, dans son acte, dans son sujet. Voir, c'est toujours une opération du sujet, donc une opération refendue, inquiétée, agitée, ouverte ${ }^{22}$. » Et la présence du témoin, parce qu'elle invite à inventer de nouvelles formes de restitution du visible et de l'événement, force par le retrait à toucher à un autre imaginaire, à prendre place tout en prenant la mesure d'autres images possibles. L'image du témoin - par le détour qu'il offre sur l'immédiateté aveuglante

\footnotetext{
${ }^{21}$ Jacques Rancière, Le Spectateur émancipé, Paris, La Fabrique, 2008, p. 18.

${ }^{22}$ Georges Didi-Huberman, Ce que nous voyons, ce qui nous regarde, Paris, Minuit, 1992, p. 51.
} 
du présent - est une image sans intention, saisie d'une impression qui n'est pas encore pensée, image «pensive » donc que Jacques Rancière définit comme étant celle qui casse l'évidence du sensible parce qu'elle «recèle de la pensée non-pensée, une pensée qui n'est pas assignable à l'intention de celui qui la voit sans qu'il la lie à un objet déterminé ${ }^{23}$. »

\title{
Le regard oblique
}

\author{
« D'ailleurs il n'y a rien à voir là-dedans. »
} Arthur Rimbaud, Enfance.

On pourrait convoquer nombre de pièces ou de projets travaillant autour de la forme du témoignage, s'appuyant sur lui dans le temps de l'élaboration - par le recours à des entretiens, à des interviews, à des enquêtes sociales, ethnologiques ou journalistiques - mais également dans le temps de la représentation - par le recours aux témoins eux-mêmes, à ces « experts du quotidien » qui font la spécificité des spectacles de Rimini Protokoll, ${ }^{24}$ par la présence sur scène de rôles de journaliste, d'enquêteur, ou de figures empruntées au réel (personnalités politiques, médiatiques, historiques, etc.) : depuis une dizaine d'années, en effet, les scènes contemporaines, via le prisme du témoignage, l'introduction d'une temporalité propre à l'enquête et la multiplication de dispositifs de déposition, d'observation ou de restitution, sont marquées par une attention documentaire, répétant et prolongeant une sensibilité à l'œuvre dans d'autres formes artistiques comme la bande-dessinée, le roman, le cinéma ou encore les arts plastiques. Cet effet de documentarisation des arts de la scène n'est pas sans lien avec le retour répété de la figure du témoin, que celui-ci soit utilisé comme matière (à écriture), comme signe (du réel) ou comme protocole (de création). Le témoignage déploie l'espace d'une dispersion et on peut voir en lui le moyen d'aller saisir l'événement là où il est encore « polycéphale » et discontinu : si l'évènement, "c'est ce qui passe ici et là ${ }^{25}$ ", le témoin reste encore ce « vivant qui passe ${ }^{26} »$, manière et moyen d'être en lui et de s'y tenir.

Plutôt que de me livrer à une étude qui ne pourrait prétendre à l'exhaustivité des cas, tant les expériences et les démarches sont nombreuses à être traversées par une tentation documentaire que trahit la présence du témoin, je souhaiterais revenir sur cet appétit pour le réel, ce goût pour l'authentique et l'enchevêtrement des catégories de la fiction et du documentaire dont témoignent l'évolution des scènes contemporaines des vingt dernières années $^{27}$. La multiplication d'ouvrages théoriques récents consacrés à cette question est

\footnotetext{
${ }^{23}$ Jacques Rancière, Le spectateur émancipé, Paris, La Fabrique, 2008, p. 115.

24 Pour plus de précision sur la méthode de travail de Rimini Protokoll voir l'article de Marie Bader, « L'élaboration de Sicherheitskonferenz : un exemple de la méthode de Rimini Protokoll », Agôn [En ligne], Points de vue \& perspectives, mis à jour le : 19/10/2010, URL : http://agon.ens-lyon.fr/index.php?id=1221.

${ }^{25}$ Michel Foucault, Leçons sur la volonté de savoir, Paris, Seuil/Gallimard, coll. " Hautes Études », 2011, p. 187. «Un événement, c'est toujours une dispersion ; une multiplicité. C'est ce qui passe ici et là, il est polycéphale. »

26 «Un Vivant qui passe », Claude Lanzmann, 1997, 65mn, Films Aleph-Cinétévé. Entretien avec Maurice Rossel réalisé par Claude Lanzmann pendant le tournage de Shoah.

${ }^{27}$ Citons par exemple le travail réalisé Guy Alloucherie avec Base 11/19 en 2007, celui de Motus dans Alexis. Une tragédie grecque, celui de Rimini Protokoll en Allemagne, le courant du théâtre verbatim en Angleterre et le teatr.doc en Russie, le théâtre-témoin dont parle Ariane Zaytzeff à propos des Etats-Unis (Ariane Zaytzeff, «Une expérience de théâtre témoin», Agôn [En ligne], Dossiers, HS $n^{\circ} 1$ : Mettre en scène l'événement, Mémoire de l'événement : le cas du 11 septembre au théâtre, mis à jour le : 30/08/2011, URL : http://w7.ens1sh.fr/agon/index.php?id=1772). Citons aussi les démarches de Sonia Chiambretto ( Chto. Trilogie »), d'Olivia Rosenthal (Les Lois de l'hospitalité) et de Franck Smith (Guantanamo), qui ne vagabondent jamais bien loin des scènes. En écho à cette inflation du témoignage, l'actualité scientifique n'est pas en reste. On pense bien sûr au
} 
révélatrice des enjeux qui se rattachent à cette présence décisive du réel et de son double : citons, par exemple, Le Réel à l'épreuve de l'écran: essai sur le principe de réalité documentaire de François Niney, paru en 2002; Le retour du réel: situation actuelle de l'avant-garde de Hal Foster paru en 1996 aux États-Unis et en 2005 dans sa traduction française; La Bande dessinée à l'épreuve du réel, ouvrage collectif coordonné par Pierre Alban Delanoy paru en 2007 ; Le Réel à l'épreuve des arts, l'écran, la rue, la scène, ouvrage collectif coordonné par Geneviève Jolly et paru en 2008 ; Reality Hunger : A manifesto de David Shields paru en $2010^{28}$. À partir de ce relevé, incomplet et rapidement esquissé, on peut non seulement constater la diversité des champs d'investigation préoccupés par ce reflux du réel, mais aussi signaler la récurrence d'une formulation, dans l'emploi réitéré du terme d' ' épreuve », qui révèle la fascination qu'entretiennent à la fois les artistes et les théoriciens pour cette idée d'une validation de l'envers - que serait l'esthétique - par son endroit - que constituerait le réel et ses déterminations éthiques. Une telle récurrence signe aussi le retour d'une exigence qui questionne l'art dans ses vertus à dire le réel, à témoigner pour lui (aussi bien à sa place qu'à son adresse).

Mais c'est par l'écart que je souhaiterais entrer dans ce goût pour le réel. Car c'est par l'inattendu, la surprise, le déport léger que se fabrique le regard oblique, regard latéral, corollaire de cette défaite du bon point de vue qu'implique la répétition jusqu'à plus soif des mêmes images et des mêmes cadrages qui institua aussi sec l'événement " 11 septembre » en objet d'attention à l'échelle du globe. Le regard oblique est le regard imposé par le réfractaire, la posture modeste et en retrait qui permet d'entrevoir un autre visage de l'événement. Et c'est à cette écriture-là, qui se constitue au fil d'un regard humble (si l'on veut bien se rappeler aussi qu'humble, humilis, voulait dire d'abord au plus près du sol), que s'est exercé Michel Vinaver dans 11 septembre 2001 et dans bon nombre de ses pièces. Dans un petit ouvrage publié un an avant les attentats et qui reprend le texte d'une intervention faite par Michel Vinaver en juin 2000 aux Journées de Soleure, apparaît cette notion d'oblicité. Dans ce texte, intitulé La Visite du Chancelier Autrichien en Suisse, Michel Vinaver évoque notamment sa formation littéraire aux États-Unis. Il se souvient avoir été frappé par un livre, Poetry, Direct and Oblique, d'un critique et théoricien, E.M.W. Tillyard : "La thèse en était que la poésie oblique va droit au but plus sûrement que la poésie qui tire tout droit ${ }^{29}$. » Il est ensuite question d'un film, visionné tout récemment par l'auteur, le film de l'autrichienne Ruth Beckermann, À L'Est de la guerre (Jenseits des Krieges) datant de 1996, et qui reposait sur le principe suivant :

La cinéaste a installé sa caméra à l'intérieur du local qui accueillait à Vienne l'exposition de photos sur les crimes de la Wehrmacht. Dans le film on ne voit pas les photos. On voit des visiteurs. Des hommes âgés. Quand Ruth Beckermann repérait parmi les visiteurs un vieillard de plus ou moins 80 ans, elle l'interpellait : «Vous y étiez ? " Et l'homme parlait. Ou bien, elle n'avait pas besoin de faire l'approche. Un vieux voyant un autre vieux ne pouvait pas ne

colloque « Le geste de témoigner : un dispositif pour le théâtre » organisé en mars 2011 à Paris et Louvain sous la direction de George Banu, Catherine Naugrette et Jean-Pierre Sarrazac, mais on pourrait également citer le colloque «Esthétique du témoignage » organisé en mars 2004 à Caen sous la direction de Carole Dornier et Renaud Dulong, ou encore la décade de Cerisy « Témoignage et écriture de l'histoire » organisée en juillet 2001 sous la direction de Jean-François Chiantaretto et Régine Robin. La question du témoin a de toute façon été au centre de toute l'histoire du $\mathrm{XX}^{\mathrm{e}}$ siècle.

${ }^{28}$ François Niney, L'épreuve du réel à l'écran, essai sur le principe de réalité documentaire, Bruxelles, De boeck, $2^{\mathrm{e}}$ édition, 2002 ; Hal Foster, Le retour du réel: situation actuelle de l'avant-garde (1996), Yves Cantraine, Frank Pierobon et Daniel Vander Gucht (trad.), Bruxelles, La Lettre volée, 2005 ; Le Réel à l'épreuve des arts, l'écran, la rue, la scène, Geneviève Jolly (dir.), Paris, L'Harmattan, coll. « Esthétiques », 2008 ; David Shields, Reality Hunger : A Manifesto, New York, Vintage Book, 2011.

${ }^{29}$ Michel Vinaver, La Visite du chancelier autrichien en Suisse, Paris, L'Arche, 2000, p. 34. 
pas l'aborder : «Vous y étiez ? » Et Ruth Beckermann filmait leur dialogue. C'était ou bien : oui, j'y étais mais je n'ai rien vu, rien su. Ou bien : j'ai su mais je n'ai pas vu et ce n'était pas moi. Ou bien : j'ai vu mais ce n'était pas moi. Par le procédé oblique de faire entendre les dénégations, et de montrer les visages des visiteurs de l'exposition, la cinéaste fait ressortir de façon bouleversante à la fois l'horreur des crimes qui ont été commis, et le couvercle qui a été posé sur ces crimes, effaçant la mémoire dans l'esprit même des criminels revenant cependant sur le lieu du crime ${ }^{30}$.

Le regard oblique, c'est donc celui de la caméra de Ruth Bekermann qui déniche ce qui a eu lieu dans le visage de celui qui parle, ne veut pas parler ou parle mais à mots couverts. On voit naître ici la parenté avec les quelques démarches précédemment citées, à ceci près que s'ajoute ici une nouvelle modalité : celle de la parole et du témoignage, qui nous rapproche du théâtre et de ses enjeux. Car ce qui fait distorsion ici, c'est bien l'absence de l'événement, que dit le sort réservé aux photographies dans le film (on ne les voit pas - le lieu de l'exposition à lui seul fait sens puisque c'est pour ces images que les témoins s'y rendent et reviennent), mais également l'écart infime, perceptible par moments, entre ce qui est dit et ce que le visage trahit: «Tentative de prendre au mot, en même temps que sur le fait, ce qui péniblement travestit [la vérité $]^{31} »$, écrit Jacques Mandelbaum à propos du film, quand la réalisatrice note, elle, dans son journal de tournage à la date du 22 octobre 1995 : «Les visages parlent, les visages trompent ${ }^{32}$.»

Dans 11 septembre 2001, Michel Vinaver, a lui aussi abondamment recours au témoignage, non pas en faisant se rencontrer une parole et un visage comme le faisait Ruth Berckermann (montage cinématographique), mais par le biais d'une rencontre entre plusieurs objets de parole (montage musical). Cette omniprésence de la parole du témoin tient à un dispositif très particulier sur lequel repose la pièce et qui fait se confronter en permanence le dedans et le dehors de l'événement, l'espace de l'observation et celui de l'expérience. Cette forme, Michel Vinaver la doit à Jean-Sébastien Bach et à ses Passions, ainsi qu'il l'écrit dans sa note liminaire et dans les brouillons préparatoires : «S'approcher de la forme de l'oratorio, telle la Création de Haydn, ou des cantates de Bach, ou plus précisément encore, de la forme des Passions de J.S.B., combinant des parties chorales, des airs attribués à des personnages, et des récitatifs ${ }^{33}$. » Pour approcher l'événement, lui donner voix et corps, le "réfléchir [...] plutôt qu'y réfléchir ${ }^{34} »$, Michel Vinaver se livre donc à l'écriture d'une partition chorale par le prélèvement de fragments empruntés au réel et à sa rumeur - celle du ressac des médias. En dehors des voix des figures du pouvoir qu'incarnent George W. Bush, Oussama Ben Laden et Donald Rumsfeld, des voix des terroristes identifiées surtout au travers du testament de Mohammed Atta, et des voix anonymes, composées d'autant de flashs, charpie du tout-venant que fait entendre le chœur, l'événement se dispose dans la géographie éclatée de voix singulières : il y a la voix des personnes à bord des avions, celle de Madeline Sweeney, hôtesse de l'air du vol American Airline 11, celle de Todd Beaver ${ }^{35}$, passager du vol United

\footnotetext{
${ }^{30}$ Ibid., p. 35 .

31 Jacques Mandelbaum, Le Monde, 19 avril 2000, cité sur le site de la réalisatrice, url= http://www.ruthbeckermann.com/home.php?il=16\&l=fra, consulté le 14 juin 2011.

32 Ruth Beckermann, «Á l'Est de la guerre. Journal de tournage. 1995 », en ligne url= http://www.ruthbeckermann.com/home.php?il=53\&l=fra, consulté le 15/06/2011.

33 Notes inédites de Michel Vinaver, «11 septembre $2001 », 1$ et 2. Voir l'album : http://www.facebook.com/media/set/?set=a.127842253932128.21866.127829563933397

${ }^{34}$ Michel Vinaver, "Mimèsis », in Dossier de presse de September 11, 2001, mise en scène de Robert Cantarella, Paris, Théâtre de la Colline, 2006. Ce dossier de presse est téléchargeable au lien suivant: http://www.colline.fr/telecharger.php?document=262.

${ }^{35}$ On peut remarquer une légère altération du nom du passager du vol 93 d'United Airlines, en réalité prénommé Todd Beamer. Le nom est corrigé dans la mise en scène réalisée par Robert Cantarella au CalArts en 2005. Neil
} 
Airline 93 ; les voix des survivants qui ont réussi à quitter les deux tours du World Trade Center avant qu'elles ne s'effondrent, Katherine Ilachinski, Judy Wein, Nat Alamo, Richard Jacobs, Dorene Smith, John Paul DeVito, Arturo Domingo, Jan Demczur, etc. Si pour la plupart des lecteurs ou spectateurs européens, ces noms ne font pas signe vers une quelconque extratextualité, pour les américains en revanche, la plupart de ces récits, recomposés par Michel Vinaver à partir de coupures de presse, visent le réel de l'événement. Jan Demczur, par exemple, a délivré plus d'une centaine d'entretiens depuis les attentats : il a été appelé à répéter jusqu'à l'épuisement le récit de la fuite héroïque permise par le dégagement d'une percée creusée à l'aide de l'arrête métallique de son racloir. Madeline Amy Sweeney, l'hôtesse de l'air du vol American Airline 11, grâce à qui des informations sur les pirates à bord de l'appareil ont pu être transmises, est maintenant commémorée par un prix à son nom, délivré chaque 11 septembre à un résident du Massachusetts qui se serait distingué par son courage en défendant ou sauvant les vies d'autrui. Tous les témoignages ne sont pas travaillés par une portée référentielle aussi forte et certains noms se sont perdus depuis dans le gigantesque fatras des souvenirs charriés, persistant seulement avec plus de discrétion au sein du cercle restreint des proches. Il n'empêche, chacune de ces histoires ou presque est susceptible d'avoir été réellement racontée, chacun de ces noms est potentiellement celui d'un des témoins ou d'un des acteurs des attentats du 11 septembre 2001.

S'il est une façon de replacer la catastrophe à l'échelle des responsabilités individuelles en débordant la focale métonymique choisie par les médias, celle de la «souffrance du bâtiment ${ }^{36}$ » qui participe à une dépersonnalisation de l'événement, ce retour des noms propres a aussi valeur de stratégie testimoniale : ce n'est pas l'auteur, Michel Vinaver, qui montre l'événement, mais un montage de voix, assemblées par fragments, qui répètent de l'intérieur et à hauteur d'homme ce qui a eu lieu. Le témoignage est, en effet, un acte discursif singulier qui suppose, comme le pacte autobiographique, le respect d'une certification référentielle extratextuelle. Passé au filtre de la mémoire et de la machine de la narration, le témoignage est, selon Paul Ricœur, ce qui fait jonction entre l'archive et la mémoire, il « donne une suite narrative à la mémoire déclarative ${ }^{37}$.» Pourquoi Michel Vinaver insiste-t-il pour que le nom des personnages soit «entendu ou vu au même titre que les paroles prononcées $^{38} »$ ? Outre un souci de clarification dans les possibilités d'attribution des voix, ce procédé a aussi pour effet de multiplier les occurrences du nom: les noms sont d'abord énoncés sur un mode discursif - puisque les personnages se présentent presque toujours euxmêmes une première fois - puis de façon répétitive sur un mode théâtral - dans la perspective de l'auteur, en effet, l'acteur ou un élément scénographique devraient en avoir la charge ${ }^{39}$.

Un simple relevé suffit à se persuader de la récurrence du procédé d'exhibition dans le texte lui-même : "Ici Madeline Sweeney » (p. 139), "Michael Woodward à l'appareil » (p. 139), «Écoutez Lisa mon nom est Beaver / Todd Beaver» (p. 139), «Je m’appelle

Young a composé une chanson, Let's roll, en hommage à cette victime dans l'album Are You Passionate? sorti en 2002. Sa femme, en collaboration avec Ken Abraham, a publié en 2002 un ouvrage intitulé Let's Roll!: Ordinary People, Extraordinary Courage, Wheaton, Tyndale House Publishers, 2002. Autant dire que les dernières paroles de Todd (Beaver) dans 11 septembre 2001 : «Are you guys ready ? / Let's roll » (p. 23) sont elles aussi chargées de ce poids du détail qui a marqué les mémoires.

${ }^{36}$ Clément Chéroux, Diplopie. L'image photographique à l'ère des medias globalisés. Essai sur le 11 septembre 2001, op. cit., p. 24.

${ }^{37}$ Paul Ricœur, La Mémoire, l'Histoire, l'Oubli, Paris, Le Seuil, coll. « L'ordre philosophique », 2000, p. 209.

${ }^{38}$ Michel Vinaver, «Note liminaire», in 11 septembre 2001 (2002), in Théatre complet, volume 8, Paris, L'Arche Éditeur, 2003,p. 133.

39 " "Qui parle ?" Le nom des personnages doit être entendu ou vu au même titre que les paroles prononcées. » Michel Vinaver, « Note liminaire à 11 septembre 2001 », op. cit., p. 133. 
Katherine Ilachinski » (p. 151), «Je m'appelle Judy Wein» (p. 151), «Je m'appelle Nat Alamo » (p. 151), « Mon nom est Richard Jacobs» (p. 151), « Mon nom est Dorene Smith » (p. 157), « Je m'appelle John Paul DeVito» (p. 159), « Je m'appelle Arturo Domingo » (p. 159). Michel Vinaver s'invente donc une place de «témoin de témoins ${ }^{40}$ », orchestrant ainsi de l'intérieur et de l'extérieur une dramaturgie permettant de rejouer sur la scène du théâtre l'exercice de l'attestation et celui de la déposition. Quelque chose de l'excès de l'événement se dit dans l'emploi du nom propre et dans la volonté de le faire entendre, manière de rendre la parole à celui qui l'a prise en lui laissant le bénéfice de parler en son nom. Le nom a donc ici valeur d'entrée dans une perspective éthique, mais n'engage pas une démarche critique, comme il pouvait le faire dans L'Instruction de Peter Weiss, dans la pièce Rwanda 94 du Groupov, ainsi que l'analyse Olivier Neveux ${ }^{41}$, ou dans Gênes 01 de Fausto Paravidino: «Les noms propres, respectueux de chaque singularité, ont valeur d'énoncé politique, écrit Olivier Neveux à propos de Rwanda 94. Cet autre chose, neuf, redistribue nécessairement le donné. Ils s'imposent, comme une vérité, une "supplémentation" sur l'ordinaire de l'existence. Ils déchirent la fatalité ${ }^{42}$. »

Si le nom dans 11 septembre 2001 permet de restituer un événement à taille humaine - et donc de poser en creux les conditions de possibilité de responsabilités individuelles -, il n'a pas vocation à dresser la carte de responsabilités politiques, collectives ou historiques : le nom ici ne sauve pas, il a valeur de point de saillance, anfractuosité dans laquelle s'engouffre l'événement, ce qui contient et déborde la forme dramatique elle-même. Puisque témoigner, c'est ce qu'on fait d'abord en son nom, la réitération a ici, tout comme le titre de la pièce, valeur de marqueur référentiel et participe de cette « autodésignation du sujet témoignant ${ }^{43}$ »: le témoignage, c'est ce qu'il y a au bout du nom, ce qui reste quand on a dépouillé la forme de son fantasme, la manifestation de son spectacle.

Cette différence d'approche dans la valeur accordée au nom tient sans doute aussi à la position particulière d'une autre voix, commune à 11 septembre 2001 et à Rwanda 94, celle du journaliste. Dans Rwanda 94, en effet, la journaliste Bee Bee Bee emporte avec elle le spectateur dans un chemin de pensée, traversée des scénarios de l'histoire, levée des ignorances dans la mise en forme dialectique d'un doute et d'une curiosité. Le nom, de ce point de vue, est la pierre de touche d'une recherche de vérité. Il est ce sur quoi butte «la tentative méticuleuse d'organiser un savoir commun à partir d'une enquête ${ }^{44}$. " Dans 11 septembre 2001, le journaliste est, comme l'auteur, en position de témoin, rapporteur anonyme des voix de ceux qui viennent raconter. Il est la voix du dehors, celle de l'observateur : «Ici où je me tiens ici / Ground Zero je vois / Des scènes inouïes de chaos et de peur / Certains sont vivants d'autres morts certains étaient vivants ${ }^{45}$. $\gg$ Si la démarche du Groupov était résolument processuelle et historique, celle de Michel Vinaver est volontairement arrêtée, temps suspendu d'un travail notatif, arrêt sur image de cet instant où

\footnotetext{
${ }^{40}$ L'expression revient dans nombre d'écrits et d'études sur le témoignage. Citons par exemple, Annette Wieviorka, L'Ere du témoin, Paris, Plon, 1998 ; Arnaud Dulong, « Témoins de témoins », in Vivre et écrire la mémoire de la Shoah. Littérature et psychanalyse, Charlotte Wardi et Pérel Wilgowicz (dir.), Paris, Nadir, coll. « Voix », 2002 ; Jonathan Haudot, " Maus et Auschwitz: des récits testimoniaux de témoignage(s) ? », in La Bande dessinée à l'épreuve du réel, Pierre-Alban Delannoy (dir.), Paris, L'Harmattan, 2007.

${ }^{41}$ Olivier Neveux, «Les noms qui sauvent », in Edipe contemporain? Tragédie, tragique, politique, Christian Biet, Paul Vanden Berghe et Karel Vanhaesebrouck (dir.), Vic La Gardiole, L'entretemps, coll. « Champ théâtral », 2007.

${ }^{42}$ Olivier Neveux, « Les Noms qui sauvent », art. cit., p. 255.

${ }^{43}$ Paul Ricœur, La Mémoire, l'Histoire, l'Oubli, op. cit., p. 204.

${ }^{44}$ Olivier Neveux, « Les Noms qui sauvent », art. cit., p. 247.

${ }^{45}$ Michel Vinaver, 11 septembre 2001, op. cit., p. 149.
} 
l'événement a lieu, attaché au désir de saisir ce moment labile et fuyant qui nous fait passer de l'accroc à la catastrophe, temps de l'imprévisible. Dans le texte « Mimèsis » où il revient sur le projet d'écriture qui sous-tend la pièce, Michel Vinaver énonce par deux fois cet impératif de fixation: " On ne peut pas imaginer à partir de l'événement du 11 septembre parce que l'événement passe l'imagination, écrit-il. Ce que j'ai essayé de faire, c'est le fixer. » Il ajoute : «Ce qui m'a motivé, c'est le besoin de fixer l'événement hors de tout commentaire, nu dans son immédiateté. Peut-être contre l'empâtement de la mémoire, contre le travail de l'oubli ${ }^{46}$. »

Restituant l'empreinte instantanée du choc, le journaliste occupe cette place particulière du témoin des témoins qui tient au désir de Michel Vinaver de parvenir à trouver un équivalent moderne à la forme des Passions de Bach : "La forme se rapproche de celle des cantates et des oratorios, écrit-il en note, se composant d'airs (à une, deux ou trois voix), de parties chorales (qui, dans la version française, restent dans la langue originale), et de récitatifs pris en charge par un "journaliste", fonction qui peut faire penser à celle de l'évangéliste dans les Passions de J.-S. Bach ${ }^{47}$. » L'Évangéliste, en effet, est le guide au sein des oratorios ayant la Passion pour objet : il est celui qui porte le récit, l'ordonne comme narrateur, et délègue la voix aux personnages qui agissent ensuite en leur nom propre ${ }^{48}$. Le paradigme du témoin, s'il fonctionne à l'intérieur de la Passion sur le mode de la délégation des voix et des regards, est aussi constitutif de la vérité évangélique : le témoin en est le garant, l'Évangéliste écrit parce qu'il a été témoin, parce qu'il est appelé à rendre témoignage des actes du Christ pour attester

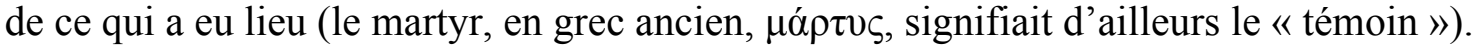

Par provocation et pour avancer de façon méthodique, on pourrait se demander ce qui différencie le recours au témoignage dans la pièce de Michel Vinaver de celui des plateaux télévisés - qu'il a par ailleurs été un des premiers à le mettre en scène (cf. À la renverse)? Est-ce qu'il y a ou non plusieurs usages du témoignage et du témoin ? Et quelle distance - si distance il y a - se glisse entre les récits de soi selon l'usage que l'on en fait ? Car tel est l'étrange privilège du témoignage que de servir aussi bien l'écart et son contraire : on trouve d'un côté des stratégies esthétiques, œuvrant à l'invention de nouveaux modes de figuration et faisant du témoignage l'opérateur d'un écart et d'une critique, ainsi que le montre le travail de Ruth Beckermann, celui de Claude Lanzmann ou celui de Michel Vinaver; on peut aussi trouver de l'autre le témoignage dévoyé en méthode d'une indécente exposition de soi, mise en scène obscène de l'intime, invitation forcenée à entrer dans la chambre des petits secrets ou instrumentalisation de cette propension à faire récit de tout, qui a notamment fait le succès de ce type d'émissions regroupé sous l'étiquette de télé-réalité et qui n'a eu de cesse de multiplier les stratégies pour «argumente[r] sur la vérité de sa relation au réel ${ }^{49}$ », ainsi que l'écrit François Josse. Il ajoute : "C'est essentiellement à travers le témoignage que la télévision de la fin du $\mathrm{XX}^{\mathrm{e}}$ siècle a prétendu atteindre une vérité. Certains y ont même vu les prémices de l'authenticité ${ }^{50}$.»

Si l'espace théâtral et le plateau télévisé sont tous deux des espaces publics, des lieux où la parole intime vient donc faire effraction, quelles sont les stratégies éthiques et esthétiques qui permettent au témoin d'être autre chose qu'un cas choisi pour l'exemple, un officiant de la

\footnotetext{
${ }^{46}$ Michel Vinaver, « Mimèsis », op. cit.

${ }^{47}$ Michel Vinaver, « Note liminaire à 11 septembre 2001 », op. cit., p. 133.

${ }^{48}$ «Le récit de l'action y est confié à un témoin, l'Évangéliste. Ce récitant vient à point nous rappeler les liens obscurs de ce "spectacle" avec l'antique action liturgique en cours. » Rémy Stricker, Musique du baroque, Paris, NRF, Gallimard, 1968, p. 92.

${ }^{49}$ François Josse, La Télévision du quotidien, entre réalité et fiction, $2^{\mathrm{e}}$ édition, Bruxelles, Ina, De Boeck, 2003, p. 9.

${ }_{50}$ Ibid., p. 10.
} 
singularité, un prête-nom pour l'illustration et pour faire pleurer dans les chaumières ? Le partage ne s'opère pas entre d'un côté l'image télévisuelle ou cinématographique et de l'autre l'espace intact et intouché du corps de l'acteur dépouillé d'artifices. Mais il concerne ces endroits où se jouent des déplacements à l'intérieur du témoignage, quel que soit le médium employé, pour que travaillent les jointures. Á quelles conditions le témoignage - parole privée - peut-elle être entendue dans un espace public ? Que faut-il pour que ce récit soit recevable si on entend par recevable ce qui me permet à moi, spectateur, de recevoir cette parole et donc d'y trouver une place, de l'admettre autant que d'y être admis ? On ne saurait tenter ici de proposer des recettes ou de dresser le profil des justes usages du témoignage. Il s'agit seulement à travers cet article de tenter de rendre compte de quelles manières certaines démarches et certaines écritures parviennent à échapper à ce "pacte compassionnel » que Dominique Mehl définit comme une « interaction spécifique entre émission et réception »:

Du côté de l'émission, le protocole compassionnel règle une mise en scène fondée sur l'exhibition de l'individu, sa souffrance particulière, et met l'accent sur la manifestation émotionnelle et sur l'expression corporelle. Du côté de la réception, l'identification au malheureux et l'empathie avec les souffrants constituent les ressorts de l'élan compassionnel $^{51}$.

Les méthodes, les approches et les stratégies diffèrent. Contrairement à l'effet d'étouffement et de saturation qu'a pu provoquer la reprise litanique des récits de rescapés dans les journaux et à la télévision, le témoignage chez Michel Vinaver ne fait pas écran parce que c'est le fait brut et banal ${ }^{52}$ que l'auteur cherche à faire parler à travers le témoin. Le récit ne s'interpose pas entre le spectateur et l'expérience esthétique, déportant l'éthique du côté des seuls affects, transformant l'image en exhibition. À la différence des témoignages hérissés de confidences et d'aveux qu'il nous a été possible d'entendre à l'époque et encore aujourd'hui, le récit des témoins dans 11 septembre 2001 s'en tient au factuel : il donne à saisir les strates de l'événement, ses vitesses et ses bifurcations, composant le mode d'un présent antérieur qui, par le détour, interroge l'avenir. La parole du témoin chez Michel Vinaver est biseautée, taillée sur la pointe du fait. Tout au plus se risque-t-elle à l'intime et à la confidence dans la dernière intervention: lancée finale, dégagement par l'anecdote que porte la fiction. Car le dernier témoignage, celui de la jeune femme anonyme sauvée du désastre par l'indigestion de son fils, est le seul récit construit de toutes pièces par l'auteur ${ }^{53}$.

Dans une perspective très différente, Rachid Ouramdane, chorégraphe associé au Théâtre de Gennevilliers et au projet D'un 11 septembre à l'autre, s'essaye lui aussi à ce regard oblique et à l'invention de conditions de recevabilité du témoignage dans un travail qui se propose depuis plusieurs années de traverser les enjeux esthétiques et éthiques de ce qu'il nomme lui-même une «poétique du témoignage ${ }^{54}$ ». Son avant-dernier spectacle, Des Témoins ordinaires (2009), plaçait le témoignage au cœur du processus de création, dans le but d'aborder un sujet pourtant délicat, celui de la torture - point limite où le langage se dérobe. Le titre du spectacle faisait à lui seul office de préambule, interrogeant le spectateur sur son rôle et sur la nature des paroles qu'on allait lui donner à entendre. Autre prolongement

\footnotetext{
${ }^{51}$ Dominique Mehl, La Télévision de l'intimité, Paris, Seuil, 1996, p. 212. Citée par Annette Wieviorka, L’Ère du témoin, op. cit., p. 179.

52 « JOURNALISTE. Voici qu'à présent la mémoire se met en orbite autour des petites choses / Le seau en métal de Jan Demczur » Michel Vinaver, 11 septembre 2001, op. cit., p. 169.

${ }^{53}$ Michel Vinaver, «Le Théâtre comme objet fractal », entretien avec Jean-Loup Rivière, in Michel Vinaver, sous la direction de Jean-Marie Thomasseau, Europe, n 924, 2006, p. 131.

${ }^{54}$ Voir notamment le site de Rachid Ouramdane, L'A - lieu de réflexion sur nos identités contemporaines, où il évoque le projet de son prochain spectacle et cette récurrence de la «poétique du témoignage »: http://www.rachidouramdane.com/index.php?id=792
} 
à un titre qui fut d'abord choisit pour rabattre l'attente du spectaculaire, que pouvait provoquer un tel sujet, sur l'ordinaire de la déposition ${ }^{55}$, le témoin ordinaire est également une expression qu'on retrouve dans le vocabulaire juridique de différents pays (Canada, Rwanda, etc.). Elle permet de désigner un individu témoin ou victime, appelé à comparaître au tribunal et dont le récit et l'expérience doivent être mis au service de l'entreprise de manifestation de la vérité à laquelle est vouée la procédure judiciaire. Si le témoin ordinaire ne se signale que par une expérience singulière, le témoin expert, lui, est sollicité en raison de son expertise professionnelle. L'autre différence essentielle qui les sépare l'un l'autre est la possibilité qu'a le témoin expert de se prononcer sur des questions hypothétiques, d'émettre un avis ou une opinion, quand le témoin ordinaire ne peut, lui, que témoigner de ce qu'il a vu, entendu ou vécu. Et c'est précisément ce que refuse Rachid Ouramdane : choisissant de réaliser un geste contraire à celui de Michel Vinaver - tant du point de vue de la démarche que du point de vue de ce qu'il attendait du récit - le chorégraphe a réalisé lui-même une série d'entretiens où le réel référentiel est comme absorbé par la question de ce que peut être le récit de l'expérience: le fait se dérobe, les références géographiques et temporelles se dissolvent, les voix, elles, resteront anonymes et parfois sans visage. Plutôt que de tenter de saisir un événement, Rachid Ouramdane plonge, lui, dans le singulier des histoires individuelles, point intime de l'humain où l'événement n'existe plus que comme expérience particulière. Située en amont du témoignage, la pièce s'interroge sur ce qui le rend possible :

Quelle est la place occupée par chacun - celui qui parle et celui qui reçoit ? [...] En face de ces témoignages, on se demande : jusqu'où peut-on faire récit de ces expériences-là, sachant que c'est une expérience inimaginable ? L'inimaginable, c'est l'endroit qui sépare la personne qui a connu la torture de celle qui ne l'a pas connue. On touche à l'écart entre l'imaginaire et l'expérience vécue. Du coup, quel imaginaire peut-on solliciter, atteindre et travailler - sur scène par exemple ? Est-ce que le témoignage le plus immédiat - avec son caractère spontané, véridique - permet de faire récit, $\operatorname{transmission}^{56}$ ?

Et c'est ce sur quoi reviennent les témoignages eux-mêmes, qui font retour de façon lancinante tout au long du spectacle, en voix off ou par le biais d'écrans placés au sol que dressent et couchent les danseurs. Les premières minutes sont à cette image. Plongée dans le noir, la salle est tendue dans l'écoute de cette parole qui filtre à travers les haut-parleurs :

C'est difficile à dire, pas parce que c'est émotionnellement difficile - ça je pense que c'est acceptable, on peut aller au-delà, mais c'est juste parce que les mots pour le dire sont difficiles à trouver. C'est un souvenir qui déborde de ce que les mots sont prévus pour exprimer. Si on arrive à le dire, et si on arrive à le dire à quelqu'un que ça intéresse, je crois qu'il subsiste encore la peur que la personne ne le croie pas. Et même si elle le croit qu'elle ne sache pas quoi en faire. C'est un souvenir dont je ne sais pas quoi faire et dont je crois que les autres ne peuvent pas savoir quoi faire ${ }^{57}$.

Comme le Rimbaud de Enfance II, on pourrait ajouter par laconisme ou par provocation : « D'ailleurs il n'y a rien à voir là-dedans », tant le spectacle choisit d'entrer dans les voix et dans leur récit par la lenteur et la fragilité. Les deux lignes, celle des voix sans corps et celle

\footnotetext{
${ }^{55}$ Rachid Ouramdane, «Poétiques du témoignage», Agôn [En ligne], Mémoire de l'événement : le cas du 11 septembre au théâtre, HS n ${ }^{\circ} 1$ : Mettre en scène l'événement, Dossiers, mis à jour le : 30/08/2011, URL : http://w7.ens-lsh.fr/agon/index.php?id=1779

${ }^{56}$ Rachid Ouramdane, entretien avec Gilles Amalvi, réalisé pour le festival d'Automne à Paris, disponible en ligne url=http://www.festival-automne.com/Publish/evenement/274/BibleOuramdane.pdf, consulté le 18/06/2011. Voir également l'entretien avec Rachid Ouramdane, «Poétiques du témoignage», Agôn [En ligne], Mémoire de l'événement : le cas du 11 septembre au théâtre, HS $n^{\circ} 1$ : Mettre en scène l'événement, Dossiers, mis à jour le : 30/08/2011, URL : http://w7.ens-lsh.fr/agon/index.php?id=1779

${ }^{57}$ Premier témoignage qui ouvre Des Témoins ordinaires.
} 
des corps sans voix des cinq interprètes se répondent depuis deux points contraires : l'un qui par le récit façonne l'imaginaire, l'autre qui par le dépouillement du plateau le sollicite autrement, par le retrait et par l'invention d'un autre mode de figuration. Jamais les rapports de domination, de violence, de maltraitance, d'humiliation ne se donnent à voir sous des traits mimétiques ou réalistes - se devinant seulement dans la tenue d'une posture intenable à laquelle le corps s'est prêté, disposition du corps insoutenable qui glisse et se défait avant même qu'on ait pu s'en inquiéter ou en prendre la mesure. Cette tension dans la lenteur est ce qui permet non pas de combler "l'écart entre l'imaginaire et l'expérience vécue », mais de l'aborder autrement que comme un fossé ou comme un miroir, une façon de le restituer - il faut lui faire une place - mais en lui inventant un autre visage. Comme Michel Vinaver qui, dans 11 septembre 2001, composait une restitution sonore et vocale de l'événement (le bruit du crash des avions réinventant le punctum de l'événement), Rachid Ouramdane travaille à la levée d'un autre imaginaire : «Ce qui m'intéresse, c'est de décliner le témoignage sous toutes ses formes : de l'ultra-réalité à l'autofiction, de l'ultra-justesse jusqu'au malentendu. C'est par cette tension qu'on peut éveiller chez le spectateur un regard critique, un sens du soupçon ou de la distance. C'est une façon de l'amener à prendre ses distances par rapport à cette tentation de la fascination ${ }^{58}$. » Prenant à rebours son goût pour l'individu et l'accentuation des particularités des interprètes, le chorégraphe s'est efforcé ici de gommer les singularités de chacun, œuvrant à un effacement des signes pour « que chaque histoire singulière converge vers un écho collectif, pour faire progressivement ressortir le caractère ordinaire de ces témoignages ${ }^{59}$. " Et c'est la tension entre ces deux pôles, celui du plateau et celui des voix, qui interpelle le spectateur en l'invitant à faire retour sur ce qu'il vient de percevoir: comme le «Et maintenant » qui clôt la pièce de Michel Vinaver et fait résonner au moment du seuil la question de la responsabilité présente, Des Témoins ordinaires ose passer la main.

Si le goût de la torsion du vocabulaire nous occupait un peu, on pourrait donc dire qu'il n'y a de témoignage recevable - ou plutôt envisageable dans une perspective non seulement éthique mais également esthétique - que dès lors qu'en lui-même s'opèrent les conditions d'un passage de témoin, entendu à la fois comme possibilité d'une transmission et brèche ouverte dans la circulation d'une mémoire. L'autre point que met en avant la confrontation de ces deux démarches, c'est la latitude des rapports possibles au témoignage : autant chez Michel Vinaver le témoignage fonctionne comme un substitut du fait, une allégation du réel jusque dans la parole ; autant chez Rachid Ouramdane, il est traversé par des lignes de fiction, matière en suspens par rapport à l'Histoire. C'est sur ces différentes approches du témoignage que je souhaiterais revenir dans la suite de cet article, manière de questionner d'abord ce désir d'aller trouver, par le bais du recours au témoin, quelque chose du réel encore en persistance (pour le dire vite, le témoignage comme document du réel - approche objectiviste), pour ensuite détailler les écarts à l'œuvre dans le discours, interroger ces échappées qui travaillent la matière (pour le dire vite encore, le témoignage comme fiction de soi - approche pragmatique).

\footnotetext{
${ }^{58}$ Rachid Ouramdane, «Poétiques du témoignage», Agôn [En ligne], Mémoire de l'événement : le cas du 11 septembre au théâtre, HS $n^{\circ} 1$ : Mettre en scène l'événement, Dossiers, mis à jour le : 30/08/2011, URL : http://w7.ens-lsh.fr/agon/index.php?id=1779

${ }^{59}$ Rachid Ouramdane, entretien avec Gilles Amalvi, op. cit.
} 


\section{No ideas but in things}

«Pas d'idée sinon dans les choses. » Le mot est connu : il est de William Carlos Williams, et Jacques Roubaud, dans un article intitulé « La tentative objectiviste », publié dans la Revue de Littérature Générale, en fait l'axiome de Charles Reznikoff, grande figure de l'objectivisme poétique américain aux côtés de Louis Zukofsky, Carl Rakosi et Georges Oppen. Parce qu'il l'a traduite, Jacques Roubaud traite ici d'une œuvre en particulier, Testimony: The United States (1885-1915) : Recitative, œuvre composée de quatre recueils parus entre 1965 et 1978. L'enchâssement des titres délivre à lui seul les perspectives esthétiques qui guident une démarche qui emprunte à la poésie objectiviste ses ambitions (l'effacement du sujet-poète) et ses outils (le prélèvement et le cut-up mais appuyé ici sur une sélection rigoureuse): l'œuvre tire sa matière d'archives des tribunaux américains par l'isolement de fragments de témoignage (Testimony), qui sont ensuite transformés en une matière sonore de vers non-rimés (Recitative), permettant ainsi de dessiner, à traits rapides, une saisie instantanée des expressions fugaces qu'emprunte le réel, et qui ainsi composées en une matière discontinue forment comme un portrait de l'Amérique à différentes périodes (The United States - 1885-1915). Dans cette deuxième opération, celle de la mise en vers, se joue une part déterminante du geste poétique, paradoxalement parce qu'elle a vocation à faire oublier et le poète et la poésie. Jacques Roubaud y revient longuement : « Le type de vers est un vers non compté, non rimé, non contaminé de pentamètre iambique, un vers libre raisonnable, pas très jazzé. Ce choix est adéquat. Il doit laisser invisible au regard immédiat le fait même, considérable, de la mise en vers, du passage à la poésie ${ }^{60}$. " $S$ 'il est question ici de Testimony, la remarque vaut aussi pour Holocauste, un autre texte de Charles Reznikoff, publié en 1975 et mis en scène par Claude Régy en 1995, long poème en douze sections réalisé lui aussi à partir de témoignages, empruntés cette fois aux archives du procès de Nuremberg et à des enregistrements du procès Eichmann. Et toujours, c'est à l'effacement du geste poétique (« invisible au regard immédiat») que le vers libre doit tendre : mise en œuvre d'une disparition, permettant d'offrir au lecteur le réel tel qu'en lui-même enfin. Le poète doit travailler à se rendre invisible - il subsiste seulement dans ce désir de pointer vers le monde : « La poésie présente l'objet afin de susciter la sensation. Elle doit être très précise sur l'objet et réticente à l'émotion ${ }^{61} »$, explique Charles Reznikoff à Jean-Paul Auxeméry en citant un poète chinois du $\mathrm{XI}^{\mathrm{e}}$ siècle.

Pourquoi convoquer la poésie américaine objectiviste pour essayer de dresser ce portrait du témoin sur les scènes contemporaines ? Pourquoi s'en aller outre-Atlantique et dans un terrain somme toute assez éloigné du drame pour détailler une démarche qu'on pourrait fort bien trouver à l'œuvre dans des formes plus directement théâtrales ? Précisément parce que ce n'est que dans la confrontation de ces trois pratiques - celle de la poésie objectiviste, celle du théâtre documentaire, et celle du retour du témoin aujourd'hui - qu'on peut saisir le sens des déplacements et des altérations, des retours et des répétitions qui se jouent dans ces rapports entre le réel et son esthétisation, entre l'objectif et le subjectif, entre le document et le témoin.

\footnotetext{
${ }^{60}$ Jacques Roubaud, «La Tentative objectiviste », in Revue de Littérature Générale, n³6, 96/2, Olivier Cadiot et Pierre Alféri (dir.), Paris, P.O.L., 1996, non paginé.

${ }^{61}$ Charles Reznikoff, « Nommer, nommer, toujours nommer. Entretien avec Charles Reznikoff », in Holocauste, Jean-Paul Auxeméry (trad.), Paris, Prétexte, 2007, p. 149.
} 
M'intéresse ici la possibilité de lire dans le geste de Reznikoff, dans ce désir d'atteindre à l'effacement au nom d'une forme d'authenticité reconduite de l'expérience du réel, un point extrême à l'intérieur d'un arc dans l'usage du témoignage conçu comme document, arc qui comprendrait à son autre bord le travail réalisé par Peter Weiss dans L'Instruction. Outre le recours au témoignage, entendu comme matière prélevée dans le réel par le biais d'un savant découpage, d'un recours à des notes de procès, à des comptes-rendus d'audience, quelque chose dans l'ambition d'une saisie du réel et de ses conditions de présentation rapproche ces deux auteurs : dans cet usage du témoignage-document, on trouverait donc d'un côté Peter Weiss et son " théâtre du compte-rendu », qui présente l'objectivité « comme un concept dont une puissance au pouvoir fait usage afin d'excuser ses actes ${ }^{62} »$, de l'autre Charles Reznikoff qui entend par objectiviste la démarche de quelqu'un « qui s'en tient presque à un témoignage de tribunal, qui exprime indirectement ses émotions par le choix de son sujet, et de sa musique s'il écrit en vers ${ }^{63}$. » Entre ces deux pôles, dont on radicalise ainsi les positions, pourraient trouver à se placer les usages et les recours au témoin conçu comme document sur les scènes contemporaines, selon les usages qui en sont fait et en fonction de l'importance accordée à la force (heuristique et politique) du montage. Si on placerait plus volontiers le travail de Michel Vinaver dans les abords de Charles Reznikoff - pour le souci porté sur l'effacement du point de vue - Michel Vinaver et Peter Weiss ont, en revanche, en commun une référence à La Divine Comédie de Dante, l'un pour la portée des voix et le rythme des chants qu'il a pu y trouver, l'autre pour la récurrence d'une formule (« Je m'appelle», « Mon nom est »), qui nous rappelle que le témoignage est toujours déjà l'œuvre d'un mort, la voix donnée à ce qui a commencé à disparaître. Au-delà de cette référence commune, l'un cherche à comprendre dans l'agencement des «blocs de témoignage ${ }^{64}$ » ce que l'autre se propose de fixer: "Réfléchir l'événement plutôt qu'y réfléchir ${ }^{65}$." Tous se retrouvent cependant à l'endroit de la forme ou plutôt à l'endroit de ce qui pourrait être la forme d'un effacement : le vers non-rimé, forme qui se donne à voir (le déroulé de la page et des retours à la ligne) et se retire d'un même mouvement (pureté et simplicité du dispositif de délégation de parole dans lequel se coulent les voix), pour " permettre aux événements de parler par eux-mêmes ${ }^{66}$ », ou offrir, dans le cas de Weiss, un " "schéma modèle" des événements actuels ${ }^{67}$ »

Dans les trois cas se retrouve également la question de l'authenticité et des stratégies qui lui sont attachées (il n'y a d'authentique que ce qui est certifié comme tel - du fromage au jean en passant par le document, le tableau ou le paysage) : le réel se trouve derrière la porte et chacun se demande comment prouver qu'il est bien là. On est donc loin de l'ambition naturaliste ou de la recherche nostalgique d'un réalisme pur et dur, il s'agit au contraire d'être au service d'un effet (l'effet de vérité ${ }^{68}$ ) et de travailler corrélativement à l'inscription d'une

\footnotetext{
${ }^{62}$ Peter Weiss, « Notes sur le théâtre documentaire », in Discours sur le genèse et le déroulement de la très longue guerre de libération du Vietnam illustrant la nécessité de la lutte armée des opprimés contre leurs oppresseurs, Jean Baudrillard (trad.), Paris, Seuil, 1968, p. 7 et p. 12.

${ }^{63}$ Charles Reznikoff, « Nommer, nommer, toujours nommer. Entretien avec Charles Reznikoff», in Holocauste, op. cit., p. 152.

${ }^{64}$ Paul Gray, «A living World. An interview with Peter Weiss » in Tulane Drama Review, New York, vol. 11, $\mathrm{n}^{\circ} 1,1966$, p. 108 cité par Denis Bablet, «L’instruction de Peter Weiss », in Les Voies de la création théâtrale, $\mathrm{n}^{\circ} 2,1970$, p. 165.

${ }^{65}$ Michel Vinaver, « Mimesis », op. cit.

${ }^{66}$ Paul Auster, "L'instant décisif », in L'Art de la faim, CEuvres romanesque et autres textes, vol. 2, Pierre Furlan (trad.), Arles, Actes Sud, 1999, p. 515.

${ }^{67}$ Peter Weiss, « Notes sur le théâtre documentaire », op. cit., p. 11.

${ }^{68}$ On distinguera « l'effet de réel » défini par Roland Barthes de « l'effet de vérité » défini par Carlo Ginzburg dans un article «Ekphrasis and quotation» (Tijdschrift voor filosofie, $\mathrm{n}^{\circ}$ 1, Leuven, Hoger instituut voor Wijsbegeerte, mars 1988) dont Béranger Boulay résume ainsi les enjeux : « Mettant en particulier l'accent sur les éléments textuels qui donnent de l'autorité et du crédit au discours de l'historien et permettent d'emporter
} 
nouvelle forme de réception (ce « nous » observateur, témoin, juge, auditeur devant qui les voix viennent à parler). Là où les positions diffèrent, c'est dans la façon de concevoir l'inscription du tiers et dans ce que chacun entend par « réel» ou par " événement». Car le réel n'a pas le même visage selon qu'on le place dans les choses, le grand bazar de la matière et des faits, ou qu'on le voit opérer dans les stratégies de pouvoir qui l'instituent comme tel. Robert Kramer, cité par François Niney dans L'Épreuve du réel à l'écran (2000), précisait ainsi : «La définition de la réalité est une construction politique. [...] Le pouvoir, c'est la possibilité de définir ce qui est réel ${ }^{69}$. $»$ L'écart est de taille entre la cafetière, le quignon, le grain de la lumière qui croît et la gestion des autoroutes, des travailleurs clandestins et des sans-papiers qui poireautent aux frontières. Éminente prérogative du réel que d'être ce qu'on voit et ce qu'on manque, ce qui interpelle et ce qui s'oublie. Autrement dit, si l'ambition commune est bien de mettre en partage le réel, de le restituer, les démarches diffèrent à l'intérieur de l'usage du témoignage comme document, du point de vue notamment de l'effet recherché entre d'un côté des écritures plutôt constatatives ou notatives et de l'autre des processus plus franchement critiques et dénonciateurs.

Dans l'un et l'autre cas, cependant, le témoignage fonctionne comme une stratégie d'authentification. Le témoin apporte une plus-value à ce qui désormais n'est plus, il est ce point intact entre la persistance et l'effacement, parce que c'est à lui que le réel confie sa répétition: le témoignage n'est jamais une conclusion, il n'existe que d'être répété, description reprise et réitérée de l'expérience intacte. Charles Reznikoff insiste sur cette parenté qui relie de son point de vue le «témoignage du tribunal et le témoignage du poète $^{70} \gg$ :

Ainsi, supposez que vous soyez témoin d'un homicide par imprudence, vous ne pouvez venir à la barre et dire : « Cet homme a commis une imprudence. » Ça c'est une conclusion. Ce que vous devez faire c'est raconter comment cet homme a agi. S'est-il arrêté avant de franchir le croisement? A-t-il regardé ? Dans ce cas-ci, c'est le jury qui décide, dans l'autre ce sont les lecteurs qui décident de ce que vous leur racontez en tant que poète ${ }^{71}$.

Difficile en lisant ce conseil en forme d'anecdote de ne pas penser à «la scène de la rue $^{72} »$, que Brecht érige en modèle pour une scène de théâtre épique. On se contentera seulement de pointer du doigt la piste épique (l'acteur témoin du personnage, de lui-même et de ce qui se joue), les prolongements qu'elle appelle étant trop conséquents pour trouver une place ici. Ce qu'il faut noter en revanche, c'est l'inclination rapide de chacun à assimiler le témoignage au réel, la déposition à la reconstitution: le témoignage présente cette vertu d'offrir des prises sensibles à l'imagination et de convoquer différemment celui qui l'entend,

l'adhésion du lecteur, Ginzburg oppose l'historiographie ancienne, qui cherchait surtout à "mettre sous les yeux", et l'historiographie savante qui, elle, met entre guillemets [...], passage du régime historiographique de l'evidentia, qui donne à "voir", à celui de l'evidence, terme qui, en anglais, peut signifier "preuve" ou "témoignage". » Bérenger Boulay, «Effets de présence et effets de vérité dans l'historiographie », in Écrire l'histoire, Littérature, $\mathrm{n}^{\circ}$ 159, Paris, Armand Colin, mars 2010, p. 26. C'est sur l'effet de vérité plus encore que sur l'effet de réel que jouent des pièces qui ont recours aux écritures du témoignage ainsi que nous le verrons en étudiant plus précisément Occupe-toi du bébé de Denis Kelly dans la suite de cette étude.

${ }^{69}$ Robert Kramer, cité par François Niney, L'épreuve du réel à l'écran, essai sur le principe de réalité documentaire, Bruxelles, De boeck, $2^{\mathrm{e}}$ édition, 2002, p. 242.

${ }^{70}$ Charles Reznikoff, « Nommer, nommer, toujours nommer. Entretien avec Charles Reznikoff», in Holocauste, op. cit., p. 152.

${ }^{71}$ Ibidem.

${ }^{72}$ Bertolt Brecht, «La scène de la rue - modèle type d'une scène de théâtre épique », in Ecrits sur le théâtre, Paris, Gallimard, Pléiade, 2000. 
spectateur, juré, auditeur, etc., parce qu'il donne l'illusion d'un retour du passé possible même si différé, en somme d'une persistance du fait.

Dans L'Ére du témoin ${ }^{73}$ (1998), Annette Wievorka détaille ces points de passage qui ont permis l'extension du régime du témoignage, entrée dans l'ère du témoin que signe le procès Eichmann en 1965, et qui passe par l'assimilation de plus en plus fréquente du témoin à l'événement, du récit du fait au fait lui-même. Alors que le procès de Nuremberg avait convoqué les témoins non pas «pour émouvoir les juges ou le public présent au tribunal, mais essentiellement pour confirmer, commenter, développer le contenu des documents écrits ${ }^{74} »$, pour le procès Eichmann, le procureur Hausner adosse le procès dans son entier à la présence des témoins et à leur récit : il ne suffit pas de rendre justice, il faut rendre sensible la réalité de ce qui fut vécu. Autrement dit, pour Hausner :

Le seul moyen de faire toucher du doigt la vérité était d'appeler les survivants à la barre en aussi grand nombre que le cadre du procès pouvait l'admettre et de demander à chacun un menu fragment de ce qu'il avait vu et de ce qu'il avait vécu. Le récit d'un certain enchaînement de circonstances fait par un seul témoin est suffisamment tangible pour être visualisé. Mises bout à bout, les dépositions successives de gens dissemblables, ayant vécu des expériences différentes, donneraient une image suffisamment éloquente pour être enregistrée. Ainsi espérais-je donner au fantôme du passé une dimension de plus, celle du réel $^{75}$

Le témoin devient donc très vite l'émissaire d'une parole où le fait semble bien vouloir à nouveau s'incarner sous les apparences du sensible. Toujours à propos du procès Eichmann, Annette Wierviorka cite Haïm Gouri qui insiste lui aussi sur l'importance donnée à ces témoignages : «Les nombreux témoins, écrit-il, n’ont pas été cités pour accumuler de la douleur et de la colère. Ils ont témoigné pour mettre en lumière le massacre dans ses détails. [...] Ils furent l'essence du procès car ils étaient les délégués autorisés de l'holocauste. Ils étaient les faits ${ }^{76}$. " Avec le procès Eichmann, s'ouvre donc dans l'espace public une place vacante pour le témoignage, quelque chose pour cette parole habitée, qu'on veut porteuse d'une histoire et donc d'une part de l'Histoire, et qui du même coup, transforme radicalement le rapport de l'historien à l'événement en changeant les conditions de l'historiographie :

L'avènement du témoin transforme profondément à terme les conditions mêmes de l'écriture de l'histoire du génocide. Avec le procès Eichmann et l'émergence du témoin, hommemémoire attestant que le passé fut et qu'il est toujours présent, le génocide devient une succession d'expérience individuelle auxquelles le public est supposé s'identifier ${ }^{77}$.

À partir de la fin des années 70 et au cours des années 80 s'amplifie et se confirme la place du témoignage, le goût pour le récit de vie, les confessions et les petits aveux. Mai 1968 joue de ce point de vue un rôle essentiel pour le rapprochement qui s'y opère entre parole et événement. C'est ce que souligne Roland Barthes dans un article majeur, "L'Écriture de l'événement », paru en 1968 dans la revue Communications : «La distance millénaire entre l'acte et le discours, l'événement et le témoignage, s'est amincie : une nouvelle dimension de l'histoire, liée désormais immédiatement à son discours est apparue, alors que toute la

\footnotetext{
${ }^{73}$ Annette Wieviorka, L'Ère du témoin, Paris, Plon, 1998.

${ }^{74}$ Ibid., p. 95.

${ }^{75}$ Gidéon Hausner, Justice à Jérusalem. Eichmann devant ses juges, Paris, Flammarion, 1966, p. 384. Cité par Annette Wieviorka, L’Ère du témoin, op. cit., pp. 96-97.

${ }^{76}$ Haïm Gouri, La Cage de verre, Paris, Albin Michel, 1964, p. 296. Cité par Annette Wierviorka, L’Ère du témoin, op. cit., pp. 114-115

${ }^{77}$ Annette Wierviorka, L'Ère du témoin, op. cit., p. 118.
} 
"science" historique avait au contraire pour tâche de reconnaître cette distance, afin de la contrôler ${ }^{78} . »$

Le développement du témoignage vient donc répondre à un double désir : au présent, il est un outil permettant de fendre l'opacité de l'immédiat, donnant des prises singulières sur l'événement au moment où il survient (immédiateté qu'ont renforcé de manière décisive les outils de communication sociale tel que Facebook, Twitter ou les services de messagerie en ligne) ; du point de vue du passé de l'événement, il offre la garantie d'une persistance du réel dans le présent où se ménage l'oubli (que renforcent les possibilités de stockage et de diffusion permises par internet: on peut citer le cas du USC Shoah Fondation Institute, exposé par Annette Wieviorka, et qui a impulsé la collecte de 51686 témoignages de survivant de la Shoah et mis en ligne $72 \%$ d'entre eux, soit près de 37348 entretiens qui sont aujourd'hui à disposition du public par le biais d'un simple clic). Le passé et le présent de l'événement sont tous deux sauvés et comme rachetés par le recours au témoin, pont jeté entre l'intime et le collectif, l'individu et la communauté, la mémoire et l'oubli. Comme le souligne Michel Foucault, « on a là une nouvelle manière de prolonger l'actualité, de la transférer d'une époque à une autre et de l'offrir au regard, au savoir, comme si elle était encore présente ${ }^{79}$. »

À partir de l'analyse de la collecte et de de la production des témoignages des rescapés juifs du génocide, Annette Wierviorka dresse le portrait d'une société hantée par une histoiremémoire, désireuse peut-être de ne s'en tenir qu'à elle, friande du récit au point de l'ériger en injonction et en «impératif social ${ }^{80}$ » : on fait du « témoin un apôtre et un prophète, écrit-elle. [...] La connaissance viendrait ainsi de la confrontation au réel, au "vrai" : le réel du site, le réel du "vécu" du déporté ${ }^{81}$. » On voit donc ainsi poindre le risque du témoignage, un risque à la hauteur de sa vertu : s'il permet d'entrer dans la fragilité du vécu, dans la complexité et la multiplicité des expériences singulières que peut faire naître un événement, le témoignage peut également participer à un effacement des enjeux politiques, historiques et sociaux au profit d'une valorisation des seuls affects, engageant celui qui l'écoute et le reçoit à se complaire dans la seule posture empathique qui, comme le rappelle à juste titre Rachid Ouramdane, donne «bonne conscience mais [...] empêche toute autre forme de dégagement ${ }^{82}$. »

Fragment de réel emprunté au monde et mis en forme sous les aspects d'un récit, le témoignage, ainsi considéré, oscille donc entre la matière brute, l'éclat du passé et l'intrigante persistance du présent. Entrée par le concret dans l'insignifiant ou dans le remarquable, il interpelle plus encore que le document parce qu'il donne chair et corps et parce qu'alors l'Histoire s'y donne sous les auspices du singulier. La position objectiviste par rapport au témoignage va donc chercher dans le récit du fait ce qui historiquement a placé le témoignage au centre : la coïncidence supposée, prétendue, secrètement espérée peut-être - car elle offre ainsi l'espoir d'une consistance du temps - entre le récit, l'événement et le réel - sainte trinité

\footnotetext{
${ }^{78}$ Roland Barthes, «L'écriture de l'événement », in Mai 1968, La Prise de la parole, Communications, $\mathrm{N}^{\circ} 12$, Paris, Le Seuil, 1968, p. 108.

${ }^{79}$ Michel Foucault, «La Vérité et les Formes juridiques », texte n¹39, in Dits et Ecrits, Tome II, 1970-1975, Paris, Gallimard, 1994, p. 584. Première publication: Michel Foucault, «A verdade e as formas juridicas », Plinio Walter Prado Jr. (trad.), Cadernos da P.U.C., $\mathrm{n}^{\circ}$ 16, Rio de Janero, juin 1974. Première publication française partielle sous le titre « La Vérité et les Formes juridiques », in Chimères, Paris, n 10, 1990.

${ }^{80}$ Annette Wierviorka, L'Ėre du témoin, op. cit., p. 171.

${ }^{81}$ Ibidem.

${ }^{82}$ Rachid Ouramdane, «Poétiques du témoignage», Agôn [En ligne], Mémoire de l'événement : le cas du 11 septembre au théâtre, HS $n^{\circ} 1$ : Mettre en scène l'événement, Dossiers, mis à jour le : 30/08/2011, URL : http://w7.ens-lsh.fr/agon/index.php?id=1779.
} 
que l'approche plus fictionnalisante s'amuse à égrener en faisant jouer l'un contre l'autre chacun des termes. Précisément parce qu'il « fait vrai », qu'il offre les outils d'une stratégie du vrai, le témoignage peut devenir un élément de subversion des binarismes commodes qui opposent le vrai et le fictif, le réel et le fantasme, le document et la fiction.

\section{Où s'arrête le documentaire, où commence la fiction?}

« Nonfiction writers imagine. Fiction writers invent. These are fundamentally different acts, performed to different ends. Unlike a fiction reader, whose only task is to imagine, a nonfiction reader is asked to behave more deeply: to imagine, and also to believe. »

David Shields, Reality Hunger.

Loin d'être une échappée libre dans les territoires sans limites de l'imagination, le recours au témoignage permet d'interroger de l'intérieur de la forme documentaire - ou à travers les codes de ce qui se donne comme tel - les endroits de déport ou de débordement. C'est au sein du même espace esthétique que celui occupé par les pratiques qui font du témoignage un document que se pose aussi la question d'une esthétique du témoignage, interrogation qui invite à réinvestir le partage qu'instaure une dichotomie franche et confortable entre fiction et documentaire. Bien souvent, en effet, cette sensibilité documentaire donne lieu à travail de sape, à la percée de points de jonction entre l'authenticité (on vous donne le réel en pâture) et sa singerie (ce n'est pas si vrai que ça en l'air), entre le réel authentifié comme tel (on vous délivre des preuves d'existence) et le glissement vers la fiction qu'induisent par exemple le seul travail d'écriture et le passage au plateau même pour ceux qui ne jouent pas. Depuis l'ouverture de Rwanda 94 avec le témoignage de Yolande Mukagasana, rescapée du génocide et interprète de son propre rôle dans l'espace de la fiction (« Je ne suis pas comédienne, je suis une survivante du génocide au Rwanda83. »), en passant par les restitutions malmenées d'entretien ou les parodies de reconstitution qui détournent les codes de l'archive dans Tout ce qui nous reste de la révolution, c'est Simon (2010) du collectif L'Avantage du doute, jusqu'au piratage des codes de l'authenticité avec Occupe-toi du bébé (2007) de Denis Kelly, pièce de «fake verbatim» composée essentiellement de témoignages et d'interrogatoires qui se déploient comme autant d'opérateurs d'authentification au sein d'une machine purement fictionnelle, se fait jour une constante qui permet de mesurer - si besoin était de le rappeler que l'authenticité est d'abord un effet, une construction du signe en vue d'une réception, bref le fruit d'une élaboration. Autrement dit, et comme le rappelle, pour le champ spécifiquement cinématographique, François Niney, on voit se dessiner « une ligne continue (mais graduée) du documentaire à la fiction, un mélange variable de prises de vue sur le vif et de mise en scène $^{84}$. » De sorte qu'il est plus que jamais temps de s'interroger sur les marges de chacun de ces espaces qu'on croit distinguer à bon compte et sans trop de frais : "Où s'arrête le documentaire, où commence la fiction ${ }^{85}$ ? " s'interroge François Niney - et on voudrait dès lors ajouter à sa suite : à quelles stratégies ont recours les artistes qui cherchent par tous les moyens à argumenter de leur rapport au réel (à la vérité ?) et pourquoi ? Quels désirs tapis, quels songes, quels fantasmes et quelles généalogies (qu'on les invente, qu'on les refuse, qu'on les assène ou qu'on les reconstitue) viennent expliquer ce désir de plus en plus présent

\footnotetext{
83 Le Groupov, Rwanda 94, Paris, éditions théâtrales, coll. « Passages francophones », 2002, p. 15.

${ }^{84}$ François Niney, L'épreuve du réel à l'écran, essai sur le principe de réalité documentaire, op. cit., p. 115.

${ }^{85}$ Ibidem.
} 
sur les scènes contemporaines - et de manière plus général dans les pratiques artistiques et télévisuelles depuis une dizaine d'années - d'aller trouver le réel, de regagner à nouveau cette patrie, îlot qu'on voudrait croire intact, natif - comme l'homme de Rousseau est nu à l'état de nature -, où subsisterait encore quelque chose d'une prétention à toucher à un au-delà de soi, quelque chose peut-être de cette « transparence perdue ${ }^{86}$ » dont parle Umberto Eco ?

Le recours au témoignage, comme la mise en scène d'une temporalité d'enquête ${ }^{87}$ ou l'exhibition du document en tant que tel ${ }^{88}$, sont des stratégies mises en œuvre de l'intérieur pour atteindre à un effet de vérité ou d'objectivité. De telles démarches n'ont rien de nouveau, et on pourrait par exemple relire le recours à la forme du roman épistolaire sous l'angle d'une stratégie d'attestation de véridicité. Ce qui est nouveau en revanche, c'est le poids pris par l'enquête et le témoignage au sein de ces stratégies : choisir le témoin ou l'enquêteur, c'est faire le choix d'une subjectivité, d'une focale parmi tant d'autres, qui travaille - depuis l'espace de la fiction - à l'argumentation d'un rapport au réel. Exemplaire de ce point de vue est la pièce de Denis Kelly, Occupe-toi du bébé, mise en scène par Olivier Werner au théâtre de la Colline en janvier 2011 : la pièce est conduite de l'intérieur par un personnage, Kelly, qui invite successivement les protagonistes d'un événement - un double infanticide - à venir témoigner. Orchestrant son enquête à travers une série d'interviews, Kelly navigue entre les témoins : il y a Donna, d'abord condamnée à la prison à perpétuité puis, à l'issue de son procès en appel, jugée irresponsable de la mort de ses deux enfants. Il y a sa mère, guerrière politique en croisade pour sa propre campagne aux élections locales, qui se bat avec la même fougue pour faire innocenter sa fille. Il y a aussi le Dr Millard qui pense avoir mis au jour un syndrome expliquant le comportement de Donna, le syndrome de Leeman-Keatley. Il y a enfin Martin, le mari de Donna, qui refuse d'entrer dans le jeu de l'interrogatoire mais finit par accepter d'être interviewé par Kelly. Rien n'est vrai, Denis Kelly (l'auteur et non le personnage) a construit de toutes pièces ce drame social aux allures d'enquête, et pourtant tout est fait pour respecter scrupuleusement les coordonnées d'un réel qu'on serait venu prendre sur le vif: la syntaxe hésitante, boiteuse, emportée et trébuchante des témoins, la convocation des différents protagonistes appelés à délivrer leur point de vue sur l'événement tout comme le refus de certains de participer à ce jeu de dupe, le recours aux documents (lettres, projections vidéo, extraits de journal télévisé, projections de Powerpoint), jusqu'au pacte d'authenticité, exhibé comme garantie, en ouverture de la pièce : «Ce qui suit a été retranscrit mot pour mot à partir d'entretiens et de correspondances. Rien n'a été ajouté et les mots utilisés sont ceux employés, même si certaines coupes ont pu être faites. Les noms n'ont pas été changés ${ }^{89}$." Car ce que met paradoxalement en scène la pièce de Kelly, c'est bien la toute-puissance de la fiction, sa souplesse et son ouverture incroyable à toutes les formes de déterminations documentaires.

La mise en scène d'Olivier Werner redouble judicieusement ce trouble, transportant la pièce de Kelly dans l'espace d'un plateau de télévision, déportant ainsi le témoignage du côté de la confession publique, interrogeant le spectateur sur

\footnotetext{
${ }^{86}$ Umberto Eco, « La Transparence perdue », in La guerre du faux, Myriam Tanant (trad.), Paris, Grasset, 1985.

87 "L'inscription de l'enquête dans le récit permet en un sens aux historiens de retrouver une autorité fondée sur l'autopsie. » Bérenger Boulay, in « Effets de présence et effets de vérité dans l'historiographie », art. cit., p. 31.

${ }^{88} \mathrm{Cf}$. dans 11 septembre 2001 la présentation du document source se fait sur le même mode que la présentation du nom du témoin : soit de l'extérieur où la nature du document est donnée de la même façon qu'un nom de personnage («FEUILLET D'INSTRUCTION AUX TERRORISTES $\left.{ }^{88} »\right)$, soit de l'intérieur par les voix elles-mêmes (« ATTA. Ceci est mon testament $\left.{ }^{88} . »\right)$

${ }^{89}$ Denis Kelly, Occupe-toi du bébé (2007), Philippe Le Moine et Pauline Sales (trad.), Paris, L'Arche, 2010.
} 
cette obscénité compassionnelle ${ }^{90}$, qui régit une bonne part des médias dominants, en usant pendant toute la première moitié du spectacle d'une caméra dont les images sont retransmises sur un immense écran : le spectateur peut juger du même œil le réel et sa copie, suivre la preuve (le comédien) et l'épreuve (l'écran) dans le trajet de l'image qui vient fouiller le visage, scruter le détail, pousser le corps dans ses retranchements pour faire saillir les lapsus et les légers silences, les malaises ou les emportements. La mise en scène d'Olivier Werner met à nu les rituels de mise en scène de soi - créant par là-même le sentiment d'un supplément de réalité : comme la télévision qui use de l'artifice du confessionnal ou de l'affichage du dispositif (voir une caméra à l'écran crée d'emblée en sentiment de plus grande réalité : le média regagne du crédit et paradoxalement se fait oublier comme tel en affichant ses outils $^{91}$ ), il s'agissait là aussi de mettre au jour cette " prétention à parler au nom du réel $^{92} »$, dont parle François Jost, en s'immisçant par la fiction dans «l'entreprise de séduction de la télévision [qui] repose depuis quarante ans sur cette annonce, cette promesse, faite au téléspectateur ${ }^{93}$. »

Et c'est à cet endroit que la pièce interpelle : pour prendre à bras le corps la question de la vérité, et celle de nos rapports fantasmés à un réel qu'on souhaiterait voir arriver sur le plateau, authentique et intouché, Denis Kelly décide de livrer ses interrogations sous la forme d'une fiction :

Je voulais écrire sur la vérité. [...] J'avais le sentiment que la vérité était d'une certaine manière menacée dans notre vie publique. Que les choses soient fausses, cela n'avait aucune importance. Si les médias pouvaient prouver la véracité d'une information, alors elle devenait vraie. Une fois que j'avais fait l'expérience de cela, je voulais aller encore plus loin. J'ai pensé que le meilleur moyen d'écrire sur la vérité serait de mentir. J'ai donc écrit une pièce verbatim qui n'était pas vraie ${ }^{94}$.

La pièce traverse pourtant cette mince frange où le document est joué, réinventé mais participe à un effet de vérité d'autant plus troublant que les personnages ne cessent de se poser eux-mêmes la question du vrai. D'abord à travers le personnage du docteur Millard qui revient sur le sentiment partagé par chacun de «transiger avec la vérité » du fait d'un état de passivité généralisé : «On observe la famine, on est au Darfour poursuivi par les milices, on voit l'ours blanc se noyer dans l'océan Arctique, on vit dans la rue avec les mendiants, et pourtant nos systèmes politiques, sociaux ou économiques n'ont pas été conçus pour gérer de

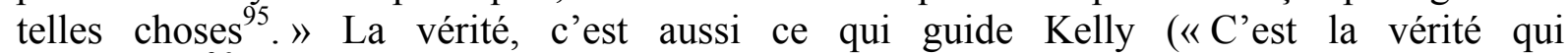
m'intéresse $\left.{ }^{96} . »\right)$, double de l'auteur dans l'espace de la fiction, délégué à l'interrogation, qui cherche à retrouver le réel en partage au-delà des points de vue singuliers défendus par

${ }^{90}$ Olivier Werner, «L'obscénité compassionnelle», Agôn [En ligne], Entretiens \& Portraits, mis à jour le : 31/08/2011, URL : http://w7.ens-lsh.fr/agon/index.php?id=1785

91 «Voici le terme du processus d'anthropomorphisation de la source de l'image : la caméra n'est plus simplement un œil au service d'une pensée qui organise le réel, elle est le prolongement d'un homme ou d'une femme qui ne témoignage pas seulement du vécu par son regard, mais dont l'apparence et l'identité ont une incidence sur la fabrication des images. » François Jost, La Télévision du quotidien, op. cit., p. 79.

${ }^{92}$ François Jost, La Télévision du quotidien, op. cit., p. 9.

${ }^{93}$ Ibidem.

${ }^{94}$ Narrative in Contemporary Drama, Dennis Kelly in conversation with Aleks Sierz, $19^{\mathrm{e}}$ Conférence annuelle de la Société Allemande pour le Théâtre Contemporain Anglophone, Paderborn, juin 2010. Extraits disponibles dans le dossier pédagogique du spectacle (url : http://www.colline.fr/telecharger.php?document=935).

${ }_{95}^{9}$ Denis Kelly, Occupe-toi du bébé, op. cit., p. 30.

${ }^{96}$ Ibid., p. 56. 
chacun des personnages. Et c'est au cours d'une confrontation entre ces personnages, tous deux préoccupés par la question du vrai, qu'a lieu un ultime échange ayant trait à la vérité :

DR MiLLARD. C'est la vérité, je vous dis que c'est la vérité mais je vous dis que ça dépend de quel point de vue, vous, je veux dire qu'il n'y a pas qu'une seule vérité, voilà ce que je voulais vous dire, je dis que - Ce que je dis c'est que tout est subjectif, il y a la vérité et puis il y a ce que les gens pensent être la vérité et cela dépend de la façon dont Et puis écoutez ne gardez pas cette partie sur la vérité, parce que vraiment c'est Je veux arriver à la vérité.

DR MILLARD. Vous voulez arriver à votre vérité, écoutez si vous ne coupez pas ça je m'en vais $^{97}$.

Autrement dit Occupe-toi du bébé use du dispositif du témoignage et de la confession pour plonger au cœur d'une question, celle de la vérité et de son histoire, à partir de l'étude des espaces où elle se forme : l'enquête et l'aveu. Ce qui intéresse Denis Kelly, c'est ce passage de la vérité envisagée comme fait (l'événement du réel) à la vérité envisagée comme effet (l'événement de la réception), du témoignage comme document au témoignage comme signe. La vraie dimension de la pièce, sous couvert d'un détournement des codes du théâtre verbatim, qui pourrait la réduire à peu de frais à un geste purement formel, est en réalité éthique : arpenter cet espace incertain où la fiction se risque à l'effet du document, où le réel semble s'engouffrer dans sa réinvention, c'est aussi revenir sur cette exigence adressée aux arts - celle de parler du monde - et de cette récurrence - sur-présence d'un réel qui n'a de cesse de se rappeler pour ce qu'il est. Manière peut-être pour l'artiste - je risque l'hypothèse de racheter sa place (l'œuvre et l'auteur trouvent ainsi à s'insérer au sein du grand tissu social du monde : parler du réel, c'est aussi y trouver sa place) et pour le spectateur de se persuader qu'il n'est pas là pour rien (il vient pour qu'on lui parle du monde - on ne saurait donc l'accuser de se carapater ailleurs, quand bien même il s'en va au théâtre). Derrière cette valorisation implicite des enjeux rattachés au documentaire et à l'actualité, que questionne Denis Kelly en mettant le spectateur face à son désir de voir tout autant qu'à son besoin de croire, ne pourrait-on pas suspecter quelque chose comme une dévalorisation implicite de la fiction, entendue comme simple simulacre ? Quelque chose comme un retour de l'angoisse Quichotte/Bovary qui ferait du document (le réel au dehors) et du récit de soi (le réel audedans) le lieu intime de la vérité préférable à toute autre forme d'ailleurs (l'irréel de la fiction) ? Reflux du vrai dans la preuve (le document chez Peter Weiss) qui trouverait son aboutissement dans l'aveu ${ }^{98}$ devenu, comme le rappelle Michel Foucault, un des «rituels majeurs dont on attend la production de vérité ${ }^{99} »$. Le développement du recours au témoignage sur les scènes contemporaines semble interroger, critiquer tout autant que prolonger ce passage à une " littérature ordonnée à la tâche infinie de faire lever au fond de soi-même, entre les mots, une vérité que la forme même de l'aveu fait miroiter comme inaccessible ${ }^{100}$. »

La fiction détournerait du monde et dans un temps où le réel, lui-même, semble échapper, farci qu'il est de simulacres, c'est plutôt vers lui, vers le tangible de l'intime et du document qu'il faudrait donc se tourner pour en saisir la pâte bien épaisse. Le témoignage apparaîtrait donc comme la possibilité d'une parole authentique, promesse d'une restitution sincère du

\footnotetext{
${ }^{97}$ Ibid., p. 74.

${ }^{98}$ C'est aussi l'hypothèse que propose François Jost à partir d'une étude des séries américaine contemporaines dans De quoi les séries américaines sont-elles le symptôme ?, CNRS éditions, coll. « Débats », 2011.

${ }^{99}$ Michel Foucault, Histoire de la sexualité, vol. I, La Volonté de savoir, Paris, Gallimard, coll. « Tell », 1976, p. 78.

${ }^{100}$ Ibid., p. 603.
} 
monde. Car, s'il est toujours susceptible d'être remis en cause du point de vue de ce qui s'y dit (fiabilité fragile du témoignage qui rend, notamment, son usage juridique et historique délicat), il apporte néanmoins la certitude d'une parole incarnée, et donc potentiellement sincère (l'attente implicite de sincérité et le devoir de vérité fondent, au moins en droit, tout échange verbal): de même que dans les années quatre-vingt-dix, on voit apparaître à la télévision, « un médiateur verbal devant la caméra, qui soumettra le visuel à la loi de sa profession : reporter ${ }^{101} »$, de même la figure du témoin, comme celle de l'enquêteur d'ailleurs qui lui aussi devient de plus en plus présent - au moins en creux dans les formes d'écriture -, permettent de retrouver « une autorité fondée sur l'autopsie ${ }^{102}$ »: comme l'écrit François Jost, « montrer, c'est témoigner ${ }^{103}$ ». Paradoxalement, en effet, plus la subjectivité s'affiche, plus le médium se donne à voir pour ce qu'il est, et plus on veut croire à un sentiment de réel ou de sincérité ( $c f$. tournage à l'épaule dans les films de fiction qui permet de reconstituer un effet documentaire). L'accent se déporte de la véridicité de l'énoncé (le document et ce qu'il contient) sur la vérité de la source d'énonciation (le témoin et sa prise de parole).

\section{Conclusion}

Événement de l'événement, le témoignage et son impression sont toujours clandestinité d'une présence: franchise d'un visage ou d'un récit qui ménage son propre horizon d'intelligibilité. Brèche ouverte dans le réel qui permet de le saisir là où il n'est plus ou n'est pas encore tout à fait, le témoignage semble offrir des prises sur ce qui par définition échappe : l'événement, évidence du «c'est ça » qui peut laisser sans voix. La récurrence du témoignage sur les scènes contemporaines n'est certainement pas guidée par les mêmes désirs et les mêmes raisons. On l'a vu, les pratiques et les usages sont aussi variés qu'inattendus : du témoignage conçu comme équivalent du fait au témoignage comme levier de forçage des limites qui assignent la fiction à résidence, la distance est grande - comme elle l'est entre la perspective notative et celle critique et dénonciatrice. Il semblerait pourtant qu'au-delà de cette diversité se fasse jour une question, qui se donne à lire sous des visages masqués, celle de la vérité. Loin d'être un concept universel, transhistorique, débordant toute rupture sociale, philosophique, ou politique, la vérité a elle aussi une histoire - ainsi que s'est attaché à le démontrer Michel Foucault tout au long de son œuvre. Et la figure de la vérité aujourd'hui, après avoir été celle de l'épreuve, puis celle du document, semble bien être aujourd'hui celle du témoin et celle de l'enquête, celle de la confession et celle de l'interrogation.

Comme l'écrit Clément Rosset, «le réel est toujours ici, mais n'apparaît jamais qu'ailleurs : il faut donc regarder ailleurs si l'on veut l'apercevoir ${ }^{104} . »$ Et le témoignage offre l'occasion d'entrer par l'oblique et le déport - le moyen d'apercevoir ce qui est ici, en regardant ailleurs. Attention comme portée corollairement sur l'essentiel, ainsi que le suggère Dubuffet qui pointe, lui aussi, cette nécessité d'un regard oblique, d'une attention transversale. Il faudrait pour voir saisir « l'objet vu sans grande attention ${ }^{105} »$, celui resté flou

\footnotetext{
${ }^{101}$ François Jost, La Télévision du quotidien, op. cit., p. 78.

102 Bérenger Boulay, «Effets de présence et effets de vérité dans l'historiographie », in Écrire l'histoire, Littérature, $\mathrm{n}^{\circ}$ 159, Paris, Armand Colin, mars 2010, p. 31.

${ }^{103}$ François Jost, La Télévision du quotidien, op. cit., p. 79.

${ }^{104}$ Clément Rosset, Le réel. Traité sur l'idiotie, Minuit, 2004, p. 124.

105 Jean Dubuffet, Prospectus et tous écrits suivants, volume II, Paris, Gallimard, 1967, cité par Michel Vinaver, « Mes appétits », in Michel Vinaver, Europe, n 924, Avril 2006, pp. 156.
} 
dans la périphérie du champ de vision. Et tel est précisément ce que permet aussi le théâtre, quand il s'immisce dans les usages du témoignage : l'invention d'une nouvelle distance, jeu ou battement entre le porte-parole et la prise de parole, distance à l'intérieur du témoignage qui permet le passage de témoin. Le caractère indiciel de la parole du témoin fait qu'elle nous est donnée tout autant que refusée, offerte par le corps (la parole s'incarne) et niée par ce même corps (qui donc ici me parle ?).

On pourrait s'interroger sur la carence qui travaille aujourd'hui notre société et notre époque pour qu'elle soit devenue si réceptive au témoignage. Serait-ce donc que notre humanité est devenue si pauvre en homme pour avoir besoin de l'assoir à toutes les tables?

À propos de 1'auteur :

Agrégée de Lettres modernes, doctorante en études théâtrales (E.N.S. de Lyon), co-rédactrice en chef de la revue Agôn, Barbara Métais-Chastanier enseigne actuellement à l'Université Paul Valéry - Montpellier 3.

Pour citer ce document :

Barbara Métais-Chastanier, «Témoigner pour le réel», Agôn [En ligne], Dossiers, HS n ${ }^{\circ} 1$ : Mettre en scène l'événement, Mémoire de l'événement : le cas du 11 septembre au théâtre, mis à jour le : 06/09/2011, URL : http://agon.ens-lyon.fr/index.php?id=1787. 